\title{
Screening Libraries of Amphiphilic Janus Dendrimers Based on Natural Phenolic Acids to Discover Monodisperse Unilamellar Dendrimersomes
}

Irene Buzzacchera, ${ }^{\dagger,}, \|$ Qi Xiao, ${ }^{\dagger,}{ }^{\circ}$ Hong Han ${ }^{\dagger}$ Khosrow Rahimi, ${ }^{\ddagger}, \perp$ Shangda Li, ${ }^{\dagger}$ Nina Yu. Kostina, ${ }^{\ddagger, \perp}$

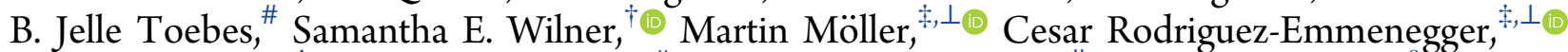

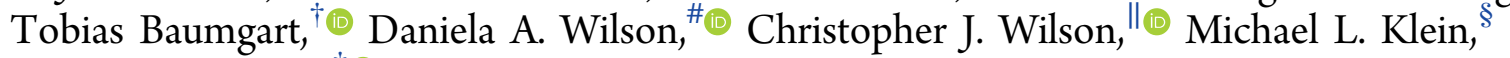
and Virgil Percec* $*+\infty$

${ }^{\dagger}$ Roy \& Diana Vagelos Laboratories, Department of Chemistry, University of Pennsylvania, Philadelphia, Pennsylvania 19104-6323, United States

${ }^{\ddagger}$ DWI-Leibniz Institute for Interactive Materials, RWTH Aachen University, 52074 Aachen, Germany

${ }$ NovioSense B.V., Transistorweg 5, 6534 AT Nijmegen, The Netherlands

${ }^{\S}$ Institute of Computational Molecular Science, Temple University, Philadelphia, Pennsylvania 19122, United States

${ }^{\perp}$ Institute of Technical and Macromolecular Chemistry, RWTH Aachen University, 52074 Aachen, Germany

\#Institute of Molecules and Materials, Radboud University, Heyendaalseweg 135, 6525 AJ Nijmegen, The Netherlands

Supporting Information

ABSTRACT: Natural, including plant, and synthetic phenolic acids are employed as building blocks for the synthesis of constitutional isomeric libraries of self-assembling dendrons and dendrimers that are the simplest examples of programmed synthetic macromolecules. Amphiphilic Janus dendrimers are synthesized from a diversity of building blocks including natural phenolic acids. They self-assemble in water or buffer into vesicular dendrimersomes employed as biological membrane mimics, hybrid and synthetic cells. These dendrimersomes are predominantly uni- or multilamellar vesicles with size and polydispersity that is predicted by their primary structure. However, in numerous cases, unilamellar dendrimersomes completely free of multilamellar assemblies are desirable. Here, we report the synthesis and structural analysis of a library containing 13 amphiphilic Janus dendrimers containing linear and branched alkyl chains on their hydrophobic part. They were prepared by an optimized iterative modular synthesis starting from natural phenolic acids. Monodisperse dendrimersomes were prepared by injection and giant polydisperse by hydration. Both were structurally characterized to select the molecular design principles that provide unilamellar dendrimersomes in higher yields and shorter reaction times than under previously used reaction conditions. These dendrimersomes are expected to provide important tools for synthetic cell biology, encapsulation, and delivery.

\section{INTRODUCTION}

Natural, including plant, $\mathrm{AB}_{n}$ phenolic acids are the building blocks employed by our laboratory for the iterative synthesis of constitutional isomeric self-assembling dendrons and dendrimers as the simplest class of Programmed Synthetic Macromolecules. ${ }^{1-12}$ Phenolic acids are naturally occurring aromatic compounds having phenol and carboxylic acid functionalities. $^{1-7}$ Our laboratory elaborated the concept of Programmed Synthetic Macromolecules, which employs biological principles to achieve a function. Specifically, the first biological principles that involve primary-secondary-tertiary-quaternary structure were employed to create a desired function. Programmed Synthetic Macromolecules are monodisperse self-assembling macromolecules with precise primary structure that includes composition, sequence, and stereochemistry. They undergo, when desired, folding into secondary and tertiary structures and self-organization into quaternary structures that are responsible for the creation of a defined function. When the periodic and quasi-periodic assemblies studied exhibit internal order, their structural and retrostructural analysis allows the elucidation of their structure and the elucidation of the mechanism of hierarchical supramolecular structure formation by a combination of methods including $\mathrm{X}$-ray diffraction together with simulation of oriented fiber diffractograms via molecular models, reconstructed electron-density

Special Issue: The Rational Design of Multifunctional RenewableResourced Materials

Received: September 19, 2018

Revised: October 21, 2018

Published: October 25, 2018 


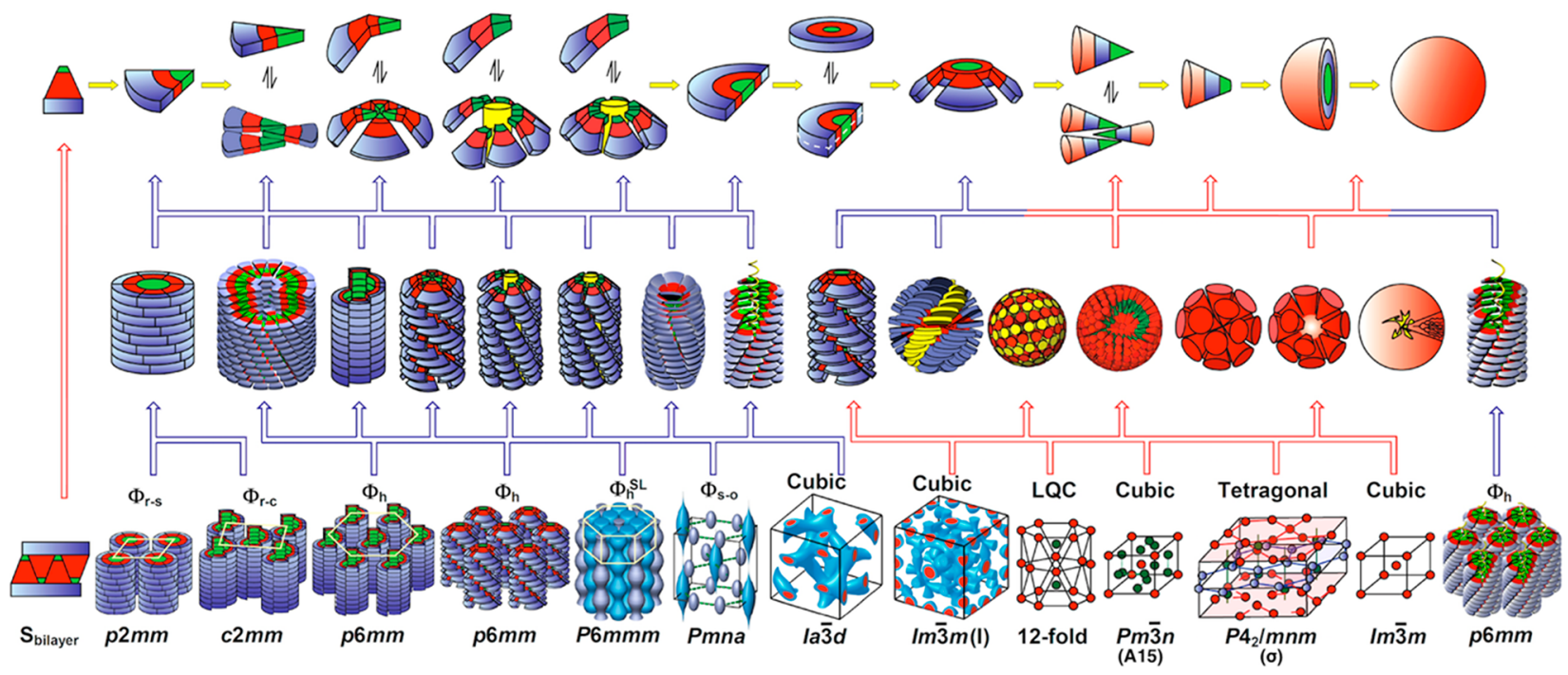

Figure 1. Accelerated synthesis of constitutional isomeric libraries of quasi-equivalent self-assembling dendrons and dendrimers and analysis of their quaternary, tertiary, and secondary structures via structural and retrostructural analysis of their periodic and quasi-periodic assemblies with internal order. ${ }^{8,9,11,29}$ Adapted with permission from ref 29. Copyright 2009, American Chemical Society.

maps, electron diffraction, transmission electron microscopy (TEM), cryo-TEM, circular dichroism, and molecular dynamics simulations (Figure 1).

The methodologies elaborated in our laboratory for structural and retrostructural analysis were inspired by the pioneering work of Watson, Crick, Klug, and others ${ }^{13-15}$ in the field of structural biology and transplanted into the field of macromolecular self-assembly. ${ }^{16-18}$ Many of the classic problems from structural biology were solved with this combination of diffraction tools. In the same way, by investigating nine generational $^{17-32}$ and one deconstruction ${ }^{33}$ libraries, our laboratory discovered soft matter quasicrystals and Frank-Kasper phases of supramolecular dendrons and dendrimers, ${ }^{17,18,34-38}$ mediated changes in the chemical reactivity and chemoselectivity, ${ }^{20,39-42}$ and discovered self-repairing supramolecular electronics, ${ }^{43-45}$ mimics of transmembrane protein channels analogous to aquaporin, ${ }^{46-54}$ molecular machines, ${ }^{55-59}$ chiral supramolecular columns and chiral supramolecular spheres, ${ }^{60-63}$ thermodynamically controlled crystallization, ${ }^{64-66}$ deracemization in the crystal state, ${ }^{67}$ a double helix that disregards chirality, ${ }^{68}$ and solid-state orientational memory effects. ${ }^{69-71}$

However, the entire machinery of life including nucleic acids, proteins, photosynthesis, and so forth is compartmentalized, assembled, and functions inside a cell, the membrane of which is the least understood biological system. The main reason for this limited understanding is that cells and cell membranes are dynamic fluid assemblies that cannot be investigated by the diffraction methodology elaborated as described above. ${ }^{72-76}$ Therefore, a fundamental question is how can one generate Programmed Synthetic Macromolecules that produce biological membrane mimics without the help of diffraction methods. A typical biological membrane is a fluidic bilayer structure coassembled from phospholipids, cholesterol, transmembrane proteins, glycolipids, glycoproteins, and other components. Once the components of a biological membranes are removed, the host phospholipids alone form vesicles named liposomes that are unstable for long time (Figure 2). ${ }^{77}$ Stealth liposomes, containing some phospholipids conjugated to polyethylene glycol or other water-soluble polymers, ${ }^{78-80}$ and amphiphilic block copolymers (their vesicles are named polymersomes), ${ }^{81}$ have been used to overcome the stability problem of phospholipid liposomes (Figure 2). However, both stealth liposomes and polymersomes are prepared by complex methods, are polydisperse, and cannot be conjugated with multiple functional groups. In addition, polymersomes have a thicker bilayer than either liposomes or biological membranes.

Our laboratory entered the field of biological membrane mimics by discovering that amphiphilic Janus dendrimers (JDs) made from constitutional isomeric $\mathrm{AB}_{2}$ and $\mathrm{AB}_{3}$ natural phenolic acids self-assemble, by injection from ethanol into water or buffer, in stable and monodisperse uni- and multilamellar onionlike (Figure 3) vesicles named dendrimersomes (DSs). ${ }^{83-99}$

The dimensions and physical properties of DSs can be predicted when they are prepared by injection but not when they are assembled by hydration. ${ }^{85}$ Their bilayer is similar in thickness to that of biological membranes, ${ }^{85}$ and unlike phospholipids and block copolymer amphiphiles, they can be functionalized with multiple components. Our laboratory next provided models for glycan-decorated cell membranes. Previously, glycopolymers, glycodendrimers, and glycoliposomes obtained by complex coassembly were the only models for biological glycans (Figure 2). Screening libraries of amphiphilic Janus glycodendrimers (JGDs) with various primary structures containing plant, bacterial, and human carbohydrates that bind to sugar-binding proteins (lectins and galectins) led to the discovery of JGDs, which self-assemble into monodisperse uniand multilamellar (Figure 3) vesicles named glycodendrimersomes (GDSs) (Figure 2). ${ }^{87}$ Subsequently, twin-twin, singlesingle, and sequence-defined JGDs and their corresponding GDSs were synthesized. The next step was to design monodisperse sequence-defined oligomers containing carbohydrates and inert oligooxyethylene-hydrophilic fragments in their structure (Figure 4). These sequence-defined oligomers selfassemble into biological membrane mimics containing various concentrations of carbohydrates (Figure 4). ${ }^{88-95}$ With these new systems, we could ask the trivial question: What is the minimum concentration of surface carbohydrate that would allow the glycodendrimer to recognize sugar binding proteins? The shocking finding was that the ability to bind proteins increased by decreasing the concentration of carbohydrate. ${ }^{89,90}$ These 

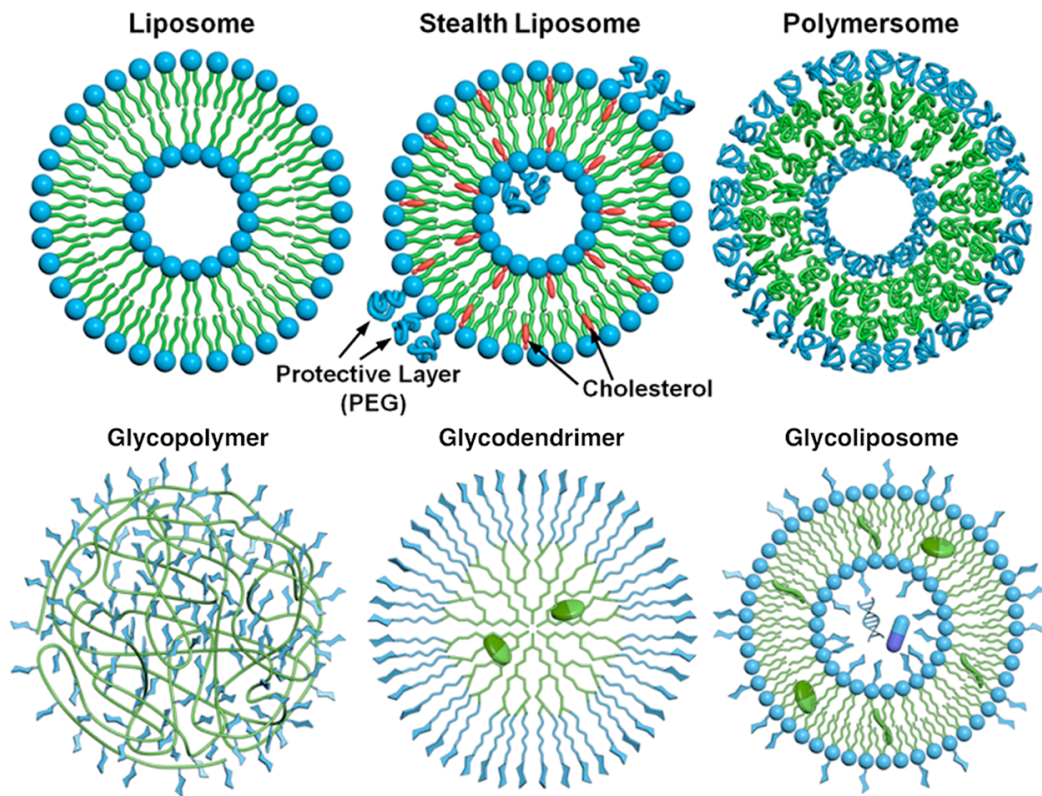

Glycodendrimer
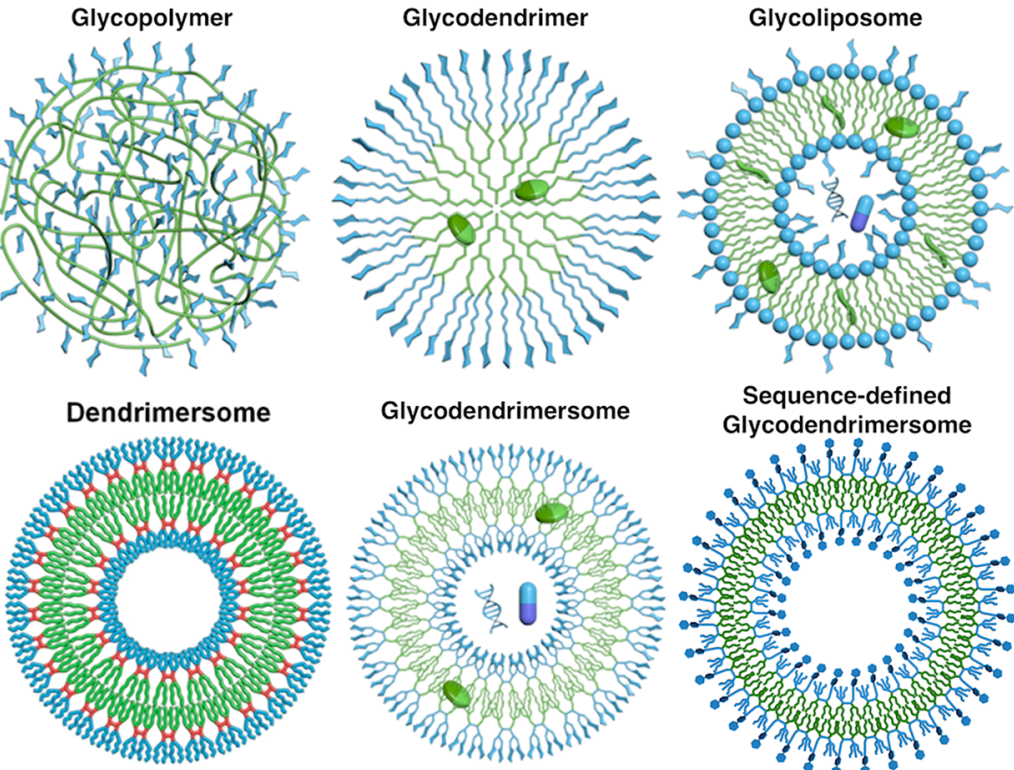

Sequence-defined

Glycodendrimersome

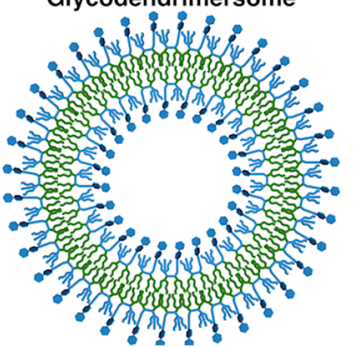

Figure 2. Structures of liposomes, stealth liposomes, polymersomes, dendrimersomes, glycopolymers, glycodendrimers, glycoliposomes, glycodendrimersomes, and sequence-defined glycodendrimersomes. Reprinted with permission from ref 84. Copyright 2014, National Academy of Sciences USA; from Springer Nature, ref 12. Copyright 2013, Springer-Verlag Berlin Heidelberg; from ref 96. Copyright 2016, National Academy of Sciences USA.

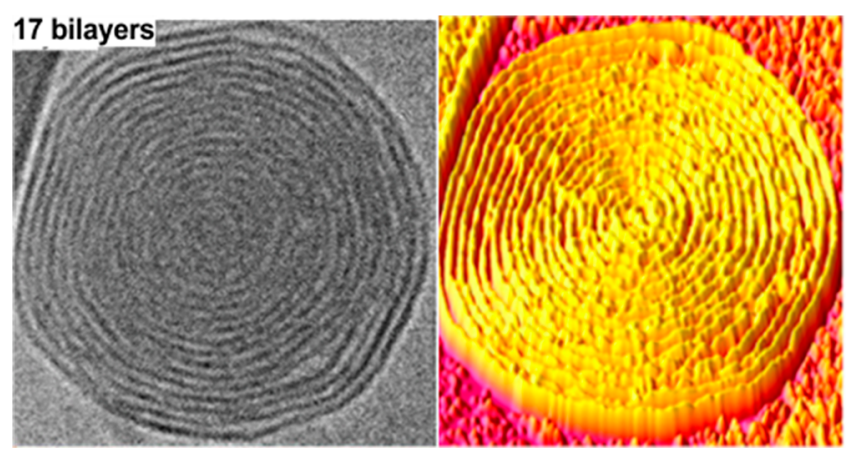

Figure 3. Multilayer onion-like dendrimersomes and glycodendrimersomes. ${ }^{84,90}$ Reprinted with permission from ref 84 . Copyright 2014, National Academy of Sciences USA.

findings indicated that, as the concentration of carbohydrate decreased, the rate constant of the recognition process increased! This observation could only be explained with a different rate constant of binding that is mediated by different carbohydrate morphologies determined by distinct supramolecular arrangements of carbohydrates on the surface of these biological membrane mimics. However, structure determination requires diffraction experiments that cannot be performed on fluid vesicles. Fluorescence microscopy is also not available because the size of dendrimersomes is below the resolution of optical microscopy. Although the methodology to answer this question is being developed, we decided to address some other fundamental problems of biological cell membrane mimics.

In previous studies, JDs with 3,5-substituted dodecyl chains were extensively used including for hybrids with DSs and bacterial membranes. ${ }^{96}$ Models for cell fusion and fission were also elaborated. ${ }^{97-99}$ Dodecyloxy chains on phenolic acid units in the hydrophobic part of the Janus dendrimers were chosen in these studies as the cell membrane mimics with similar bilayer width to the natural phospholipids that have 16 or 18 carbons in their alkyl groups. This bilayer width demonstrates that the phenolic acid fragments are part of the hydrophobic part of the bilayer. The self-assemblies from JDs containing 3,5-substituted dodecyl chains, one of the routinely employed Janus dendrimers, form mixtures of uni- and multilamellar onion-like DSs. The aim of this report is to deepen the knowledge on the size and stability of unilamellar DSs self-assembled by JDs synthesized from a fixed hydrophilic dendron and a variable hydrophobic dendron made from a phenolic acid core substituted with diverse alkyl chains of different lengths and degrees of branching. For understanding the effect of the number of carbon atoms of the linear alkyl chains or branched alkyl chain, a library containing 13 JDs was synthesized, and the factors affecting the self-assembled monodisperse unilamellar DSs were investigated and are reported here.

\section{EXPERIMENTAL SECTION}

Materials. All reagents were obtained from commercial sources and were used without purification unless otherwise stated. $\mathrm{CH}_{2} \mathrm{Cl}_{2}$ (DCM) 

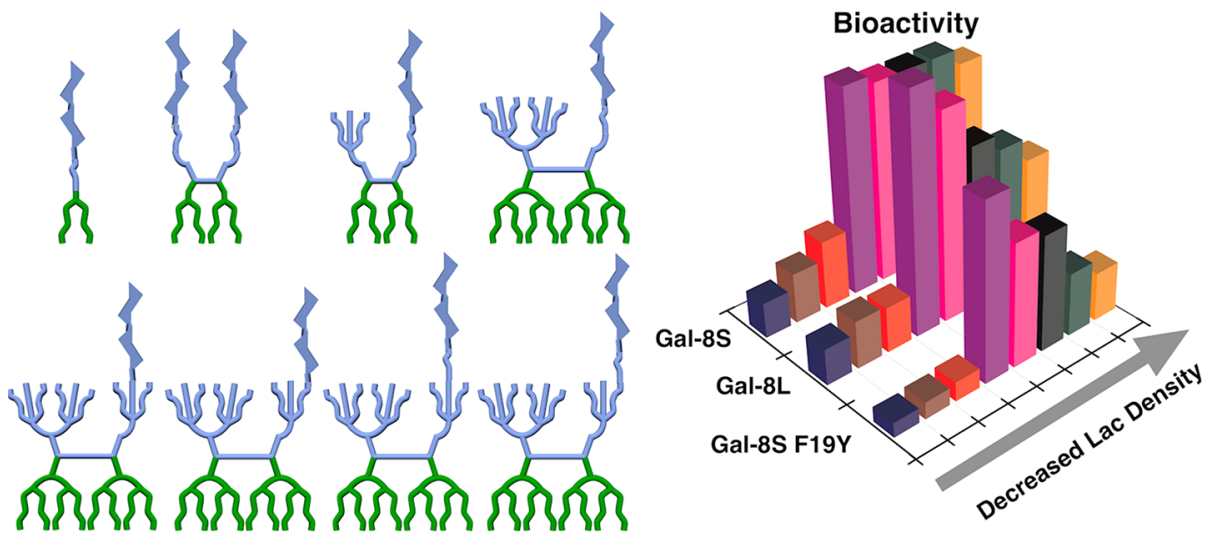

Figure 4. Sequence-defined Janus glycodendrimers self-assemble in glycodendrimersomes and their agglutination with human galectins (absorbance and rate constant $k){ }^{89}$ Reprinted with permission from ref 89. Copyright 2015, American Chemical Society.

was dried over $\mathrm{CaH}_{2}$ and freshly distilled before use. Tetrahydrofuran (THF) for preparation of DSs was distilled with $\mathrm{NaHSO}_{3}$ and 3,5-ditert-butyl-4-hydroxytoluene (BHT) and restored in te dark. Solvents and reagents were deoxygenated when necessary by purging with $\mathrm{N}_{2}$. Milli-Q water obtained from Milli-Q UV plus with resistivity of $18.2 \mathrm{M} \Omega \mathrm{cm}$ was used for the preparation of phosphate-buffered saline (PBS). PBS (1X) was obtained by dissolving $8 \mathrm{~g}$ of $\mathrm{NaCl}, 0.2 \mathrm{~g}$ of $\mathrm{KCl}$, $1.44 \mathrm{~g}$ of $\mathrm{Na}_{2} \mathrm{HPO}_{4}$, and $0.24 \mathrm{~g}$ of $\mathrm{KH}_{2} \mathrm{PO}_{4}$ in $800 \mathrm{~mL}$ of Milli-Q water adjusted to $\mathrm{pH} 7.4$ and diluted to $1000 \mathrm{~mL}$.

Techniques. ${ }^{1} \mathrm{H}$ and ${ }^{13} \mathrm{C}$ NMR spectra were recorded at 500 and $126 \mathrm{MHz}$, respectively, on a Bruker DRX $(500 \mathrm{MHz})$ NMR spectrometer. All NMR spectra were measured at $23{ }^{\circ} \mathrm{C}$ in $\mathrm{CDCl}_{3}$. Chemical shifts $(\delta)$ are reported in ppm, and coupling constants $(J)$ are reported in hertz $(\mathrm{Hz})$. The resonance multiplicities in the ${ }^{1} \mathrm{H}$ NMR spectra are described as "s" (singlet), "d" (doublet), "t" (triplet), and " $\mathrm{m}$ " (multiplet). Residual protic solvent of $\mathrm{CDCl}_{3}\left({ }^{1} \mathrm{H}, \delta 7.26 \mathrm{ppm} ;{ }^{13} \mathrm{C}\right.$, $\delta 77.16 \mathrm{ppm}$ ) or tetramethylsilane (TMS) was used as the internal reference in the ${ }^{1} \mathrm{H}$ and ${ }^{13} \mathrm{C}$ NMR spectra. Evolution of the reaction was monitored by thin-layer chromatography (TLC) using silica gel $60 \mathrm{~F}_{254^{-}}$ precoated plates (E. Merck), and compounds were visualized by UV light with a wavelength of $254 \mathrm{~nm}$. Purifications by flash column chromatography were performed using flash silica gel from Silicycle (60 $\AA, 40-63 \mu \mathrm{m}$ ) with the indicated eluent. The purity of the products was determined by a combination of TLC, and high-pressure liquid chromatography (HPLC) was carried out using a Shimadzu LC-20AD high-performance liquid chromatograph pump, PE Nelson Analytical 900 Series integration data station, Shimadzu RID-10A refractive index (RI) detector, Shimadzu SPD-10A VP (UV-vis), and three AM gel columns (a guard column, two $500 \AA, 10 \mu \mathrm{m}$ columns). THF was used as solvent at the oven temperature of $23^{\circ} \mathrm{C}$. Detection was done by UV absorbance at $254 \mathrm{~nm}$. MALDI-TOF mass spectra were performed on a PerSeptive Biosystem-Voyager-DE (Framingham, MA) mass spectrometer equipped with nitrogen laser $(337 \mathrm{~nm})$ and operating in linear mode. Internal calibration was performed using Angiotensin II and Bombesin as standards. The analytical samples were obtained by mixing the THF solution of the sample $(5-10 \mathrm{mg} / \mathrm{mL})$ with the THF solution of the matrix $(2,5$-dihydroxybenzoic acid, $10 \mathrm{mg} / \mathrm{mL})$ in a $1: 5(\mathrm{v} / \mathrm{v})$ ratio. The prepared solution of sample and matrix $(0.5 \mu \mathrm{L})$ was loaded on the MALDI plate and allowed to dry at $23^{\circ} \mathrm{C}$ before the plate was inserted into the vacuum chamber of the MALDI instrument. The laser steps and voltages applied were adjusted depending on both the molecular weight and the nature of each analyzed compound.

Preparation of Monodisperse Dendrimersomes by Injection. A stock solution was prepared by dissolving the required amount of amphiphilic JD in THF. DSs were then generated by injection of $50 \mu \mathrm{L}$ of the stock THF solution of JD into $1.00 \mathrm{~mL}$ of Milli-Q water or PBS followed by $5 \mathrm{~s}$ of vortexing. An Eppendorf Research Plus Single Channel Pipette $(10-100 \mu \mathrm{L})$ and a VWR Signature Ergonomic High Performance Single-Channel Variable Volume Pipettor (100-1000 $\mu \mathrm{L}$ ) were used for injection into Milli-Q water or PBS.
Preparation of Polydisperse Giant Dendrimersomes by Hydration. A $200 \mu \mathrm{L}(0.2 \mathrm{~mL})$ solution of JD $\left(10 \mathrm{mg} \mathrm{mL}^{-1}\right)$ in THF was deposited on the top surface of a roughened Teflon sheet $\left(1 \mathrm{~cm}^{2}\right)$ and placed in a $20 \mathrm{~mL}$ flat-bottom vial followed by evaporation of the solvent for $2 \mathrm{~h}$. The Teflon sheet was dried in vacuo for an additional $12 \mathrm{~h}$. Milli-Q water or PBS $(2.0 \mathrm{~mL})$ was added to submerge the film on the Teflon sheet, and the vial was placed in a $60{ }^{\circ} \mathrm{C}$ oven for $12 \mathrm{~h}$ for hydration. The vial containing the Teflon sheet was then mixed using a vortex mixer for $30 \mathrm{~s}$. The final concentration of JD in Milli-Q water is $1 \mathrm{mg} \mathrm{mL} L^{-1}$.

Dynamic Light Scattering (DLS). DLS for the monodisperse vesicles was performed in PBS and in Milli-Q water with a Malvern Instruments particle sizer (Zetasizer Nano S, Malvern Instruments, UK) equipped with $4 \mathrm{~mW} \mathrm{He}-\mathrm{Ne}$ laser $633 \mathrm{~nm}$ and avalanche photodiode positioned at $175^{\circ}$ to the beam and temperature-controlled cuvette holder. Instrument parameters were determined automatically along with measurement times. Experiments were performed in triplicate.

Cryogenic Transmission Electron Microscopy (Cryo-TEM). CryoTEM micrographs were taken on a Carl Zeiss Libra 120 Microscope (Oberkochen, Germany). Cryo-TEM samples were prepared by plunge freezing of an aqueous dispersion on plasma-treated lacey grids. The vitrified specimens were transferred to a Gatan-910 cryo-holder. The images were recorded at a temperature of $-170{ }^{\circ} \mathrm{C}$ with an acceleration voltage of $120 \mathrm{kV}$.

Confocal Fluorescence Microscopy. An imaging chamber $(5-10 \mu \mathrm{L})$ containing DSs was formed between two coverslips $(25 \times 25 \mathrm{~mm}$, Fisher Scientific) sealed with vacuum grease. DSs were imaged by confocal fluorescence microscopy (FluoView 300 scanning system configured on an IX81 inverted microscope platform) with a $60 \times 1.2 \mathrm{NA}$ water immersion lens (Olympus, Center Valley, PA). DSs containing 3,5C12-RhB were excited at a wavelength of $\lambda=543 \mathrm{~nm} .3,5-\mathrm{C12}-\mathrm{RhB}$ is a JD with 3,5-bis(dodecyloxy)benzoate hydrophobic dendrons conjugated to Rhodamine $\mathrm{B}(\mathrm{RhB})$ that was previously reported to coassemble with JD with red fluorescence. ${ }^{97}$ Laser intensities were adjusted so that fluorescence signal was not oversaturated. Image processing and analysis were completed with ImageJ 1.50 software.

Synthesis of Amphiphilic Janus Dendrimers. Accelerated modular synthesis of a library of Janus dendrimers is shown in Scheme 1. Compounds 1-6 and 3,5-C12-RhB were synthesized as previously reported. ${ }^{82,97}$ These alkylation conditions will later be called conventional reaction conditions.

Synthesis of Methyl 3,5-Bis(octyloxy)benzoate at Conventional Reaction Temperature and Time. ${ }^{30,32,82} 1$-Bromooctane $(5.0 \mathrm{~g}$, $26.2 \mathrm{mmol}), 3,5$-dihydroxybenzoate $(2.0 \mathrm{~g}, 11.9 \mathrm{mmol})$, and $\mathrm{K}_{2} \mathrm{CO}_{3}$ $(8.2 \mathrm{~g}, 60 \mathrm{mmol})$ were added in a $250 \mathrm{~mL}$ round-bottom flask and dissolved in $50 \mathrm{~mL}$ of $\mathrm{N}, \mathrm{N}$-dimethylformamide (DMF). The reaction was allowed to stir at $80{ }^{\circ} \mathrm{C}$ under $\mathrm{N}_{2}$ for $15 \mathrm{~h}$. The mixture was cooled at room temperature, and $100 \mathrm{~mL}$ of water was added. The aqueous layer was extracted with DCM 4 times. The organic phase was dried with $\mathrm{Na}_{2} \mathrm{SO}_{4}$, and the solvent was removed. The product was purified 
Scheme 1. Synthesis of a Library of Janus Dendrimers
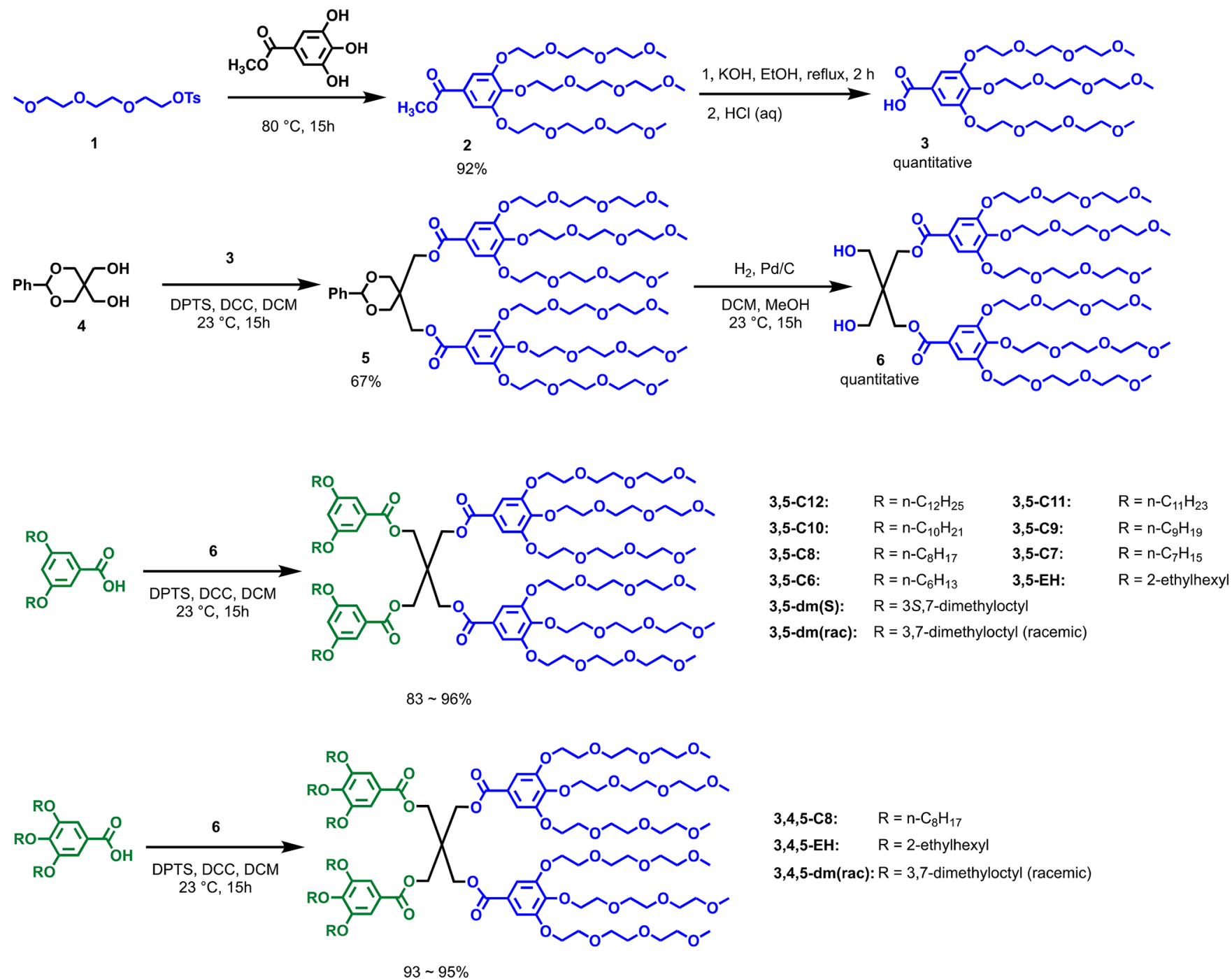

by column chromatography on silica gel with a mobile phase (hexane/ $\mathrm{DCM}=3: 1)$ to obtain the target ester. Product: 4.6 g, yield: $98 \%$.

General Optimized Procedure for Alkylation at Higher Reaction Temperature and Shorter Reaction Time. Alkyl bromide and methyl 3,5-dihydroxybenzoate or methyl 3,4,5-trihydroxybenzoate ( 400 mg) and $\mathrm{K}_{2} \mathrm{CO}_{3}$ were added in a $50 \mathrm{~mL}$ round-bottom flask. DMF $(5 \mathrm{~mL})$ was also added. The reaction was allowed to stir at $600 \mathrm{rpm}$ and $120^{\circ} \mathrm{C}$ under $\mathrm{N}_{2}$ for $3.5-6.5 \mathrm{~h}$ while monitored by conversion by TLC. The mixture was cooled at room temperature, and $30 \mathrm{~mL}$ of water was added. The aqueous layer was extracted with DCM 4 times. The organic phase was dried with $\mathrm{Na}_{2} \mathrm{SO}_{4}$, and the solvent was removed. The product was purified by column chromatography on silica gel with hexane/ethyl acetate as mobile phase to obtain the target ester.

Methyl 3,5-Bis(hexyloxy)benzoate. Reagents: 1-bromohexane (825 mg, $5.0 \mathrm{mmol}$ ), methyl 3,5-dihydroxybenzoate $(400 \mathrm{mg}$, $2.4 \mathrm{mmol})$, and $\mathrm{K}_{2} \mathrm{CO}_{3}(2.0 \mathrm{~g}, 14.4 \mathrm{mmol})$ for $4 \mathrm{~h}$. Eluent for silica column: hexane/ethyl acetate $=30: 1$. Product: $600 \mathrm{mg}$, yield: $74 \%$.

Methyl 3,5-Bis(heptyloxy)benzoate. Reagents: 1-bromoheptane (752 mg, $4.2 \mathrm{mmol}$ ), methyl 3,5-dihydroxybenzoate (336 mg, $2.0 \mathrm{mmol}$ ), and $\mathrm{K}_{2} \mathrm{CO}_{3}(1.6 \mathrm{~g}, 12 \mathrm{mmol})$ for $3.5 \mathrm{~h}$. Eluent for silica column: hexane/ethyl acetate $=30: 1$. Product: $576 \mathrm{mg}$, yield: $80 \%$.

Methyl 3,5-Bis(nonyloxy)benzoate. Reagents: 1-bromononane (1.0 g, $5 \mathrm{mmol})$, methyl 3,5-dihydroxybenzoate $(400 \mathrm{mg}, 2.4 \mathrm{mmol})$, and $\mathrm{K}_{2} \mathrm{CO}_{3}(2.0 \mathrm{~g}, 14.4 \mathrm{mmol})$ for $3.5 \mathrm{~h}$. Eluent for silica column: hexane/ethyl acetate $=30: 1$. Product: $760 \mathrm{mg}$, yield: $75 \%$.

Methyl 3,5-Bis(decyloxy)benzoate. Reagents: 1-bromodecane (930 mg, $4.2 \mathrm{mmol}$ ), methyl 3,5-dihydroxybenzoate $(336 \mathrm{mg}$,
$2.0 \mathrm{mmol}$ ), and $\mathrm{K}_{2} \mathrm{CO}_{3}(1.66 \mathrm{~g}, 12 \mathrm{mmol})$ for $3 \mathrm{~h}$. Eluent for silica column: hexane/ethyl acetate $=30: 1$. Product: $710 \mathrm{mg}$, yield: $80 \%$.

Methyl 3,5-Bis(undecyloxy)benzoate. Reagents: 1-bromoundecane (920 mg, $3.9 \mathrm{mmol}$ ), methyl 3,5-dihydroxybenzoate $(312 \mathrm{mg}$, $1.85 \mathrm{mmol})$, and $\mathrm{K}_{2} \mathrm{CO}_{3}(1.54 \mathrm{~g}, 11.1 \mathrm{mmol})$ for $4.5 \mathrm{~h}$. Eluent for silica column: hexane $/$ ethyl acetate $=30: 1$. Product: $819 \mathrm{mg}$, yield: 93\%.

Methyl 3,5-Bis(dodecyloxy)benzoate. Reagents: 1-bromododecane (933 mg, $3.8 \mathrm{mmol})$, methyl 3,5-dihydroxybenzoate $(300 \mathrm{mg}, 1.8 \mathrm{mmol})$, and $\mathrm{K}_{2} \mathrm{CO}_{3}(1.54 \mathrm{~g}, 10.6 \mathrm{mmol})$ for $4.5 \mathrm{~h}$. Eluent for silica column: hexane/ethyl acetate $=30: 1$. Product: $820 \mathrm{mg}$, yield: $91 \%$.

Methyl 3,5-Bis(2-ethylhexyloxy)benzoate. Reagents: 2-ethylhexyl bromide ( $811 \mathrm{mg}$, $4.2 \mathrm{mmol}$ ), methyl 3,5-dihydroxybenzoate (336 mg, $2.0 \mathrm{mmol}$ ), and $\mathrm{K}_{2} \mathrm{CO}_{3}(1.66 \mathrm{~g}, 12 \mathrm{mmol})$ for $4.5 \mathrm{~h}$. Eluent for silica column: hexane/ethyl acetate $=30: 1$. Product: $410 \mathrm{mg}$, yield: $50 \%$.

Methyl 3,5-Bis(3S,7-dimethyloctyloxy)benzoate. Reagents: 1-bromo-3S,7-dimethyloctane $(840 \mathrm{mg}, 3.8 \mathrm{mmol}){ }^{68}$ methyl 3,5dihydroxybenzoate $(300 \mathrm{mg}, 1.8 \mathrm{mmol})$, and $\mathrm{K}_{2} \mathrm{CO}_{3}(1.66 \mathrm{~g}, 12 \mathrm{mmol})$ for $4.5 \mathrm{~h}$. Eluent for silica column: hexane/ethyl acetate $=30: 1$. Product: $690 \mathrm{mg}$, yield: $85 \%$.

Methyl 3,5-Bis(3,7-dimethyloctyloxy)benzoate. Reagents: 1-bromo-3,7-dimethyloctane (racemic) $(840 \mathrm{mg}, 3.8 \mathrm{mmol}),{ }^{68}$ methyl 3,5-dihydroxybenzoate $(300 \mathrm{mg}, 1.8 \mathrm{mmol})$, and $\mathrm{K}_{2} \mathrm{CO}_{3}(1.66 \mathrm{~g}$, $12 \mathrm{mmol}$ ) for $4.5 \mathrm{~h}$. Eluent for silica column: hexane/ethyl acetate = 30:1. Product: $600 \mathrm{mg}$, yield: $74 \%$.

Methyl 3,4,5-Tris(octyloxy)benzoate. Reagents: 1-bromooctane $(1.0 \mathrm{~g}, 5.2 \mathrm{mmol})$, methyl 3,4,5-trihydroxybenzoate $(300 \mathrm{mg}, 1.6 \mathrm{mmol})$, 
and $\mathrm{K}_{2} \mathrm{CO}_{3}(1.8 \mathrm{~g}, 13.2 \mathrm{mmol})$ for $6.5 \mathrm{~h}$. Eluent for silica column: hexane/ethyl acetate $=30: 1$. Product: $510 \mathrm{mg}$, yield: $61 \%$.

Methyl 3,4,5-Tris(2-ethylhexyloxy)benzoate. Reagents: 2-ethylhexyl bromide (1.4 g, $7.2 \mathrm{mmol}$ ), methyl 3,4,5-trihydroxybenzoate (400 mg, $2.2 \mathrm{mmol}$ ), and $\mathrm{K}_{2} \mathrm{CO}_{3}(1.8 \mathrm{~g}, 13.2 \mathrm{mmol}$ ) for $6.5 \mathrm{~h}$. Eluent for silica column: hexane $/$ ethyl acetate $=40: 1$. Product: $510 \mathrm{mg}$, yield: $45 \%$.

Methyl 3,4,5-Tris(3,7-dimethyloctyloxy)benzoate. Reagents: 1-bromo-3,7-dimethyloctane (racemic) (1.6 g, $7.2 \mathrm{mmol}$ ), methyl 3,4,5-trihydroxybenzoate $(400 \mathrm{mg}, 2.2 \mathrm{mmol})$, and $\mathrm{K}_{2} \mathrm{CO}_{3}(1.8 \mathrm{~g}$, $13.2 \mathrm{mmol}$ ) for $6.5 \mathrm{~h}$. Eluent for silica column: hexane/ethyl acetate = 60:1. Product: $640 \mathrm{mg}$, yield: $48 \%$.

General Procedure for the Hydrolysis of Esters. The ester and $\mathrm{KOH}$ were added in a $50 \mathrm{~mL}$ round-bottom flask together with $10 \mathrm{~mL}$ of ethanol. The reaction was allowed to stir at $100^{\circ} \mathrm{C}$ for $2 \mathrm{~h}$. The mixture was evaporated under vacuum. Thirty milliliters of water $(30 \mathrm{~mL})$ was added, and the remaining mixture was cooled at $0{ }^{\circ} \mathrm{C}$. Hydrochloride acid $(2 \mathrm{M})$ was added dropwise until $\mathrm{pH}<2$. The aqueous layer was extracted with DCM 4 times. The organic phase was dried with $\mathrm{Na}_{2} \mathrm{SO}_{4}$, and the solvent was removed to obtain the target 3,5bis(alkyloxy)benzoic acid or 3,4,5-tris(alkyloxy)benzoic acid without further purification in quantitative yield.

General Procedure for Esterification for Janus Dendrimers (JDs). Compound 6, 3,5-bis(alkyloxy)benzoic acid or 3,4,5-tris(alkyloxy)benzoic acid, and 4-(dimethylamino)pyridinium 4-toluenesulfonate $(\text { DPTS })^{100}$ were mixed and dissolved in DCM $(10 \mathrm{~mL}) . N, N^{\prime}$ Dicyclohexylcarbodiimide (DCC) was added to the reaction mixture. The mixture was then allowed to stir at $23^{\circ} \mathrm{C}$ for $12 \mathrm{~h}$. The precipitate was then filtered, and the filtrate was concentrated to dryness. The crude product was further purified by column chromatography on silica gel with a mobile phase of (DCM/methanol) to yield the target JD as a colorless oily liquid.

3,5-C6. Reagents: Compound 6 (200 mg, $0.15 \mathrm{mmol})$, 3,5bis(hexyloxy)benzoic acid (103 mg, $0.32 \mathrm{mmol})$, DPTS $(88 \mathrm{mg}$, $0.30 \mathrm{mmol})$, and DCC $(124 \mathrm{mg}, 0.60 \mathrm{mmol})$. Eluent for silica column: $\mathrm{DCM} /$ methanol $=20: 1$. Product: $260 \mathrm{mg}$, yield: $89 \%$.

3,5-C7. Reagents: Compound $6(290 \mathrm{mg}, 0.20 \mathrm{mmol}), 3,5-$ bis(heptyloxy)benzoic acid (308 mg, $0.80 \mathrm{mmol}$ ), DPTS $(260 \mathrm{mg}$, $0.80 \mathrm{mmol})$, and DCC $(272 \mathrm{mg}, 1.3 \mathrm{mmol})$. Eluent for silica column: $\mathrm{DCM} /$ methanol $=20: 1$. Product: $410 \mathrm{mg}$, yield: $94 \%$.

3,5-C8. Reagents: Compound 6 (200 mg, $0.15 \mathrm{mmol}$ ), 3,5bis(octyloxy)benzoic acid (144 mg, $0.38 \mathrm{mmol})$, DPTS $(112 \mathrm{mg}$, $0.38 \mathrm{~mol})$, and DCC (124 mg, $0.60 \mathrm{mmol})$. Eluent for silica column: $\mathrm{DCM} /$ methanol $=20: 1$. Product: $255 \mathrm{mg}$, yield: $83 \%$.

3,5-C9. Reagents: Compound $6(200 \mathrm{mg}, 0.15 \mathrm{mmol})$, 3,5bis(nonyloxy)benzoic acid $(146 \mathrm{mg}, 0.36 \mathrm{mmol})$, DPTS $(132 \mathrm{mg}$, $0.45 \mathrm{mmol})$, and DCC ( $155 \mathrm{mg}, 0.75 \mathrm{mmol})$. Eluent for silica column: $\mathrm{DCM} /$ methanol $=20: 1$. Product: $278 \mathrm{mg}$, yield: $89 \%$.

3,5-C10. Reagents: Compound $6(220 \mathrm{mg}, 0.17 \mathrm{mmol}), 3,5-$ bis(decyloxy)benzoic acid (304 mg, $0.70 \mathrm{mmol})$, DPTS $(206 \mathrm{mg}$, $0.70 \mathrm{mmol})$, and DCC (210 mg, $1.0 \mathrm{mmol})$. Eluent for silica column: $\mathrm{DCM} / \mathrm{methanol}=40: 1$. Product: $322 \mathrm{mg}$, yield: $88 \%$.

3,5-C11. Reagents: Compound 6 (225 mg, $0.17 \mathrm{mmol}), 3,5-$ bis(undecyloxy)benzoic acid (199 mg, $0.43 \mathrm{mmol})$, DPTS (151 mg, $0.51 \mathrm{mmol})$, and DCC (177 mg, $0.86 \mathrm{mmol})$. Eluent for silica column: $\mathrm{DCM} /$ methanol $=20: 1$. Product: $347 \mathrm{mg}$, yield: $92 \%$.

3,5-C12. Reagents: Compound $6(370 \mathrm{mg}, 0.28 \mathrm{mmol}), 3,5-$ bis(hexyloxy)benzoic acid (274 mg, $0.56 \mathrm{mmol}$ ), DPTS (164 mg, $0.56 \mathrm{mmol})$, and DCC (206 mg, $1.0 \mathrm{mmol})$. Eluent for silica column: $\mathrm{DCM} /$ methanol $=20: 1$. Product: $530 \mathrm{mg}$, yield: $84 \%$.

3,5-EH. Reagents: Compound 6 (171 mg, $0.13 \mathrm{mmol}), 3,5$-bis(2ethylhexyloxy)benzoic acid $(200 \mathrm{mg}, 0.53 \mathrm{mmol})$, DPTS $(156 \mathrm{mg}$, $0.53 \mathrm{mmol})$, and DCC (161 mg, $0.78 \mathrm{mmol})$. Eluent for silica column: $\mathrm{DCM} /$ methanol $=40: 1$. Product: $172 \mathrm{mg}$, yield: $85 \%$.

3,5- $d m(S)$. Reagents: Compound 6 (200 mg, $0.20 \mathrm{mmol}), 3,5-$ bis(3S,7-dimethyloctyloxy)benzoic acid $(261 \mathrm{mg}, 0.6 \mathrm{mmol})$, DPTS $(117 \mathrm{mg}, 0.6 \mathrm{mmol})$, and DCC (186 mg, $0.9 \mathrm{mmol})$. Eluent for silica column: DCM/methanol = 20:1. Product: $310 \mathrm{mg}$, yield: $96 \%$.

3,5-dm(rac). Reagents: Compound $6(200 \mathrm{mg}, 0.20 \mathrm{mmol}), 3,5-$ bis(3,7-dimethyloctyloxy)benzoic acid $(261 \mathrm{mg}, 0.6 \mathrm{mmol})$, DPTS
(117 mg, $0.6 \mathrm{mmol})$, and DCC (186 mg, $0.9 \mathrm{mmol})$. Eluent for silica column: DCM/methanol = 20:1. Product: $305 \mathrm{mg}$, yield: $95 \%$.

3,4,5-C8. Reagents: Compound $6(100 \mathrm{mg}, 0.08 \mathrm{mmol}), 3,5-$ bis(octyloxy)benzoic acid (131 mg, $0.26 \mathrm{mmol}$ ), DPTS $(68 \mathrm{mg}$, $0.23 \mathrm{mmol})$, and DCC $(78 \mathrm{mg}, 0.38 \mathrm{mmol})$. Eluent for silica column: $\mathrm{DCM} /$ methanol $=20: 1$. Product: $162 \mathrm{mg}$, yield: $93 \%$.

3,4,5-EH. Reagents: Compound 6 (200 mg, $0.15 \mathrm{mmol}), 3,4,5-$ tris(2-ethylhexyloxy)benzoic acid (193 mg, $0.83 \mathrm{mmol}$ ), DPTS (132 $\mathrm{mg}, 0.45 \mathrm{mmol})$, and DCC $(155 \mathrm{mg}, 0.75 \mathrm{mmol})$. Eluent for silica column: DCM/methanol = 20:1. Product: $326 \mathrm{mg}$, yield: $95 \%$.

3,4,5-dm(rac). Reagents: Compound 6 (200 mg, $0.15 \mathrm{mmol}), 3,4,5-$ tris(3,7-dimethyloctyloxy)benzoic acid $(225 \mathrm{mg}, 0.38 \mathrm{mmol})$, DPTS $(132 \mathrm{mg}, 0.45 \mathrm{mmol})$, and DCC (155 mg, $0.75 \mathrm{mmol})$. Eluent for silica column: DCM/methanol = 20:1. Product: $365 \mathrm{mg}$, yield: $93 \%$.

Purity by HPLC, ${ }^{1} \mathrm{H} N \mathrm{NMR},{ }^{13} \mathrm{C} N \mathrm{MR}$, and MALDI-TOF for Previously Unreported Compounds. 3,5-C7. Purity (HPLC): 99\%+. ${ }^{1} \mathrm{H}$ NMR $\left(500 \mathrm{MHz}, \mathrm{CDCl}_{3}\right) \delta 7.24(\mathrm{~s}, 4 \mathrm{H}), 7.09(\mathrm{~d}, J=2.3 \mathrm{~Hz}, 4 \mathrm{H}), 6.61(\mathrm{t}, J=$ $2.3 \mathrm{~Hz}, 2 \mathrm{H}), 4.61(\mathrm{~s}, 4 \mathrm{H}), 4.58(\mathrm{~s}, 4 \mathrm{H}), 4.22(\mathrm{~m}, 4 \mathrm{H}), 4.16(\mathrm{~m}, 8 \mathrm{H})$, $3.93(\mathrm{t}, J=6.5 \mathrm{~Hz}, 8 \mathrm{H}), 3.86(\mathrm{~m}, 8 \mathrm{H}), 3.79(\mathrm{~m}, 4 \mathrm{H}), 3.71(\mathrm{~m}, 12 \mathrm{H})$, $3.64(\mathrm{~m}, 24 \mathrm{H}), 3.53(\mathrm{~m}, 12 \mathrm{H}), 3.36(\mathrm{~s}, 6 \mathrm{H}), 3.35(\mathrm{~s}, 12 \mathrm{H}), 1.76(\mathrm{~m}$, $8 \mathrm{H}), 1.44(\mathrm{~m}, 8 \mathrm{H}), 1.38-1.25(\mathrm{~m}, 24 \mathrm{H}), 0.88(\mathrm{t}, J=6.9 \mathrm{~Hz}, 12 \mathrm{H})$. ${ }^{13} \mathrm{C}$ NMR $\left(126 \mathrm{MHz}, \mathrm{CDCl}_{3}\right) \delta 165.9,165.5,160.3,152.5,143.1$, 131.1, 124.3, 109.1, 107.8, 106.7, 72.6, 72.0, 70.9, 70.8, 70.6, 69.7, 69.0, 68.4, 63.1, 63.0, 59.1, 43.4, 31.9, 29.3, 29.2, 26.1, 22.7, 14.2. MALDITOF $(m / z):[\mathrm{M}+\mathrm{Na}]^{+}$calcd for $\mathrm{C}_{103} \mathrm{H}_{168} \mathrm{O}_{36} \mathrm{Na}$, 2004; found, 2006.

3,5-C9. Purity (HPLC): 99\%+. ${ }^{1} \mathrm{H}$ NMR $\left(500 \mathrm{MHz}, \mathrm{CDCl}_{3}\right) \delta 7.24$ $(\mathrm{s}, 4 \mathrm{H}), 7.09(\mathrm{~d}, J=2.3 \mathrm{~Hz}, 4 \mathrm{H}), 6.61(\mathrm{t}, J=2.3 \mathrm{~Hz}, 2 \mathrm{H}), 4.61(\mathrm{~s}, 4 \mathrm{H})$, $4.58(\mathrm{~s}, 4 \mathrm{H}), 4.22(\mathrm{~m}, 4 \mathrm{H}), 4.17(\mathrm{~m}, 8 \mathrm{H}), 3.93(\mathrm{t}, J=6.5 \mathrm{~Hz}, 8 \mathrm{H})$, $3.86(\mathrm{~m}, 8 \mathrm{H}), 3.79(\mathrm{~m}, 4 \mathrm{H}), 3.72(\mathrm{~m}, 12 \mathrm{H}), 3.64(\mathrm{~m}, 24 \mathrm{H}), 3.53$ $(\mathrm{m}, 12 \mathrm{H}), 3.36(\mathrm{~s}, 6 \mathrm{H}), 3.35(\mathrm{~s}, 12 \mathrm{H}), 1.76(\mathrm{~m}, 8 \mathrm{H}), 1.44(\mathrm{~m}, 8 \mathrm{H})$, $1.36-1.26(\mathrm{~m}, 40 \mathrm{H}), 0.88(\mathrm{t}, J=6.9 \mathrm{~Hz}, 12 \mathrm{H}) .{ }^{13} \mathrm{C}$ NMR $(126 \mathrm{MHz}$, $\left.\mathrm{CDCl}_{3}\right) \delta 165.8,165.4,160.2,152.4,143.0,131.0,124.1,109.0,107.7$, $106.6,77.4,77.2,76.9,72.4,71.9,70.8,70.6,70.5,70.5,69.6,68.9,68.3$, 63.1, 62.9, 58.9, 43.3, 31.8, 29.5, 29.4, 29.2, 29.2, 26.0, 22.6, 14.1 . MALDI-TOF $(m / z):[\mathrm{M}+\mathrm{Na}]^{+}$calcd for $\mathrm{C}_{111} \mathrm{H}_{184} \mathrm{O}_{36} \mathrm{Na}, 2116$; found, 2116.

3,5-C10. Purity (HPLC): 98\%+. ${ }^{1} \mathrm{H}$ NMR $\left(500 \mathrm{MHz}, \mathrm{CDCl}_{3}\right)$ $\delta 7.24(\mathrm{~s}, 4 \mathrm{H}), 7.08(\mathrm{~d}, J=2.3 \mathrm{~Hz}, 4 \mathrm{H}), 6.61(\mathrm{t}, J=2.3 \mathrm{~Hz}, 2 \mathrm{H}), 4.61$ $(\mathrm{s}, 4 \mathrm{H}), 4.58(\mathrm{~s}, 4 \mathrm{H}), 4.21(\mathrm{~m}, 4 \mathrm{H}), 4.16(\mathrm{~m}, 8 \mathrm{H}), 3.92(\mathrm{t}, J=6.5 \mathrm{~Hz}$, $8 \mathrm{H}), 3.85(\mathrm{~m}, 8 \mathrm{H}), 3.79(\mathrm{~m}, 4 \mathrm{H}), 3.72(\mathrm{~m}, 12 \mathrm{H}), 3.63(\mathrm{~m}, 24 \mathrm{H}), 3.53$ $(\mathrm{m}, 12 \mathrm{H}), 3.36(\mathrm{~s}, 6 \mathrm{H}), 3.35(\mathrm{~s}, 12 \mathrm{H}), 1.76(\mathrm{~m}, 8 \mathrm{H}), 1.44(\mathrm{~m}, 8 \mathrm{H})$, $1.35-1.27(\mathrm{~m}, 48 \mathrm{H}), 0.88(\mathrm{t}, J=6.9 \mathrm{~Hz}, 12 \mathrm{H}) .{ }^{13} \mathrm{C}$ NMR $(126 \mathrm{MHz}$, $\left.\mathrm{CDCl}_{3}\right) \delta 165.7,165.3,160.2,152.3,142.9,130.9,124.1,109.0,107.6$, $106.5,72.4,71.8,70.2,70.6,70.5,70.4,69.5,68.9,68.2,63.0,62.8,58.9$, $43.3,31.8,29.5,29.3,29.2,29.1,26.0,22.6,14.0$. MALDI-TOF $(\mathrm{m} / z)$ : $[\mathrm{M}+\mathrm{Na}]^{+}$calcd for $\mathrm{C}_{115} \mathrm{H}_{192} \mathrm{O}_{36} \mathrm{Na}, 2172$; found, 2172 .

3,5-C11. Purity (HPLC): $99 \%+.{ }^{1} \mathrm{H}$ NMR $\left(500 \mathrm{MHz}, \mathrm{CDCl}_{3}\right.$ ) $\delta 7.24(\mathrm{~s}, 4 \mathrm{H}), 7.08(\mathrm{~d}, J=2.3 \mathrm{~Hz}, 4 \mathrm{H}), 6.61(\mathrm{t}, J=2.3 \mathrm{~Hz}, 2 \mathrm{H}), 4.61$ $(\mathrm{s}, 4 \mathrm{H}), 4.58(\mathrm{~s}, 4 \mathrm{H}), 4.22(\mathrm{t}, J=4.5 \mathrm{~Hz}, 4 \mathrm{H}), 4.16(\mathrm{t}, J=4.5 \mathrm{~Hz}, 8 \mathrm{H})$, $3.92(\mathrm{t}, J=6.5 \mathrm{~Hz}, 8 \mathrm{H}), 3.85(\mathrm{~m}, 8 \mathrm{H}), 3.79(\mathrm{~m}, 4 \mathrm{H}), 3.72(\mathrm{~m}, 12 \mathrm{H})$, $3.65(\mathrm{~m}, 24 \mathrm{H}), 3.52(\mathrm{~m}, 12 \mathrm{H}), 3.36(\mathrm{~s}, 6 \mathrm{H}), 3.35(\mathrm{~s}, 12 \mathrm{H}), 1.76$ $(\mathrm{m}, 8 \mathrm{H}), 1.42(\mathrm{~m}, 8 \mathrm{H}), 1.25(\mathrm{~m}, 48 \mathrm{H}), 0.87(\mathrm{t}, J=6.9 \mathrm{~Hz}, 12 \mathrm{H})$. ${ }^{13} \mathrm{C}$ NMR $\left(126 \mathrm{MHz}, \mathrm{CDCl}_{3}\right) \delta 166.0,165.6,160.4,152.6,143.2$, 131.2, 124.3, 109.2, 107.9, 106.8, 72.6, 72.1, 71.0, 70.9, 70.7, 70.7, 69.8, 69.1, 68.5, 63.2, 63.2, 59.2, 43.5, 32.1, 29.8, 29.8, 29.6, 29.5, 29.4, 26.2, 22.9, 14.3. MALDI-TOF $(m / z):[\mathrm{M}+\mathrm{K}]^{+}$calcd for $\mathrm{C}_{119} \mathrm{H}_{200} \mathrm{O}_{36} \mathrm{~K}$, 2244; found, 2245.

3,5-EH. Purity (HPLC): 99\%+. ${ }^{1} \mathrm{H}$ NMR $\left(500 \mathrm{MHz}, \mathrm{CDCl}_{3}\right) \delta 7.25$ $(\mathrm{s}, 4 \mathrm{H}), 7.11(\mathrm{~d}, J=2.3 \mathrm{~Hz}, 4 \mathrm{H}), 6.63(\mathrm{t}, J=2.3 \mathrm{~Hz}, 2 \mathrm{H}), 4.61(\mathrm{~s}, 4 \mathrm{H})$, $4.59(\mathrm{~s}, 4 \mathrm{H}), 4.22(\mathrm{~m}, 4 \mathrm{H}), 4.17(\mathrm{~m}, 8 \mathrm{H}), 3.87-3.82(\mathrm{~m}, 16 \mathrm{H}), 3.79$ $(\mathrm{m}, 4 \mathrm{H}), 3.72(\mathrm{~m}, 12 \mathrm{H}), 3.63(\mathrm{~m}, 24 \mathrm{H}), 3.53(\mathrm{~m}, 12 \mathrm{H}), 3.36(\mathrm{~s}, 6 \mathrm{H})$, $3.35(\mathrm{~s}, 12 \mathrm{H}), 1.69(\mathrm{~m}, 12 \mathrm{H}), 1.54-1.30(\mathrm{~m}, 24 \mathrm{H}), 0.94-0.88$ $(\mathrm{m}, 24 \mathrm{H}) .{ }^{13} \mathrm{C} \mathrm{NMR}\left(126 \mathrm{MHz}, \mathrm{CDCl}_{3}\right) \delta 165.9,165.5,160.5,152.4$, 143.0, 131.0, 124.2, 109.1, 107.8, 106.7, 72.5, 72.0, 70.8, 70.7, 70.6, 70.6, 69.7, 69.0, 62.9, 62.8, 59.0, 43.4, 39.4, 30.5, 29.1, 23.8, 23.1, 14.1, 11.2. MALDI-TOF $(m / z):[\mathrm{M}+\mathrm{Na}]^{+}$calcd for $\mathrm{C}_{107} \mathrm{H}_{176} \mathrm{O}_{36} \mathrm{Na}, 2160$; found, 2162.

3,5-dm(S). Purity (HPLC): 99\%+. ${ }^{1} \mathrm{H}$ NMR $\left(500 \mathrm{MHz}, \mathrm{CDCl}_{3}\right)$ $\delta 7.24(\mathrm{~s}, 4 \mathrm{H}), 7.10(\mathrm{~d}, J=2.3 \mathrm{~Hz}, 4 \mathrm{H}), 6.62(\mathrm{t}, J=2.2 \mathrm{~Hz}, 2 \mathrm{H}), 4.61$ $(\mathrm{s}, 4 \mathrm{H}), 4.59(\mathrm{~s}, 4 \mathrm{H}), 4.21(\mathrm{~m}, 4 \mathrm{H}), 4.17(\mathrm{~m}, 8 \mathrm{H}), 3.97(\mathrm{~m}, 8 \mathrm{H}), 3.86$ 
(m, $8 \mathrm{H}), 3.79(\mathrm{~m}, 4 \mathrm{H}), 3.72(\mathrm{~m}, 12 \mathrm{H}), 3.64(\mathrm{~m}, 24 \mathrm{H}), 3.53(\mathrm{~m}, 12 \mathrm{H})$, $3.36(\mathrm{~s}, 6 \mathrm{H}), 3.35(\mathrm{~s}, 12 \mathrm{H}), 1.85-1.62(\mathrm{~m}, 12 \mathrm{H}), 1.60-1.48(\mathrm{~m}, 8 \mathrm{H})$, $1.36-1.12(\mathrm{~m}, 20 \mathrm{H}), 0.93(\mathrm{~d}, J=6.6 \mathrm{~Hz}, 12 \mathrm{H}), 0.86(\mathrm{~d}, J=6.6 \mathrm{~Hz}$, $24 \mathrm{H}) .{ }^{13} \mathrm{C}$ NMR $\left(126 \mathrm{MHz}, \mathrm{CDCl}_{3}\right) \delta 165.9,165.5,160.3,152.4$, 143.0, 131.1, 124.2, 109.1, 107.8, 106.6, 72.5, 72.0, 70.8, 70.7, 70.6, 69.7, 69.0, 66.7, 62.8, 59.0, 43.4, 39.3, 37.3, 36.1, 29.8, 28.0, 24.7, 22.7, 22.6, 19.6. MALDI-TOF $(\mathrm{m} / z):[\mathrm{M}+\mathrm{Na}]^{+}$calcd for $\mathrm{C}_{115} \mathrm{H}_{192} \mathrm{O}_{36} \mathrm{Na}$, 2172; found, 2172.

3,5-dm(rac). Purity (HPLC): 99\%+. ${ }^{1} \mathrm{H}$ NMR (500 MHz, $\mathrm{CDCl}_{3}$ ) $\delta 7.25(\mathrm{~s}, 4 \mathrm{H}), 7.10(\mathrm{~d}, J=2.3 \mathrm{~Hz}, 4 \mathrm{H}), 6.62(\mathrm{t}, J=2.3 \mathrm{~Hz}, 2 \mathrm{H}), 4.61$ $(\mathrm{s}, 4 \mathrm{H}), 4.59(\mathrm{~s}, 4 \mathrm{H}), 4.21(\mathrm{~m}, 4 \mathrm{H}), 4.17(\mathrm{~m}, 8 \mathrm{H}), 3.97(\mathrm{~m}, 8 \mathrm{H}), 3.86$ $(\mathrm{m}, 8 \mathrm{H}), 3.79(\mathrm{~m}, 4 \mathrm{H}), 3.72(\mathrm{~m}, 12 \mathrm{H}), 3.66-3.62(\mathrm{~m}, 24 \mathrm{H}), 3.52$ $(\mathrm{m}, 12 \mathrm{H}), 3.36(\mathrm{~s}, 6 \mathrm{H}), 3.35(\mathrm{~s}, 12 \mathrm{H}), 1.85-1.64(\mathrm{~m}, 12 \mathrm{H}), 1.60-$ $1.48(\mathrm{~m}, 8 \mathrm{H}), 1.36-1.11(\mathrm{~m}, 20 \mathrm{H}), 0.93(\mathrm{~d}, J=6.6 \mathrm{~Hz}, 12 \mathrm{H}), 0.86$ $(\mathrm{d}, J=6.6 \mathrm{~Hz}, 24 \mathrm{H}) .{ }^{13} \mathrm{C} \mathrm{NMR}\left(126 \mathrm{MHz}, \mathrm{CDCl}_{3}\right) \delta 165.9,165.5$, 160.3, 152.5, 143.1, 131.1, 124.3, 109.2, 107.9, 106.7, 72.6, 72.0, 70.9, 70.8, 70.6, 69.7, 69.0, 66.8, 63.0, 59.1, 43.4, 39.3, 37.4, 36.2, 29.9, 28.0, 24.7, 22.8, 22.7, 19.7. MALDI-TOF $(m / z):[\mathrm{M}+\mathrm{Na}]^{+}$calcd for $\mathrm{C}_{115} \mathrm{H}_{192} \mathrm{O}_{36} \mathrm{Na}$, 2172; found, 2172.

3,4,5-EH. Purity (HPLC): 99\%+. ${ }^{1} \mathrm{H}$ NMR (500 MHz, $\left.\mathrm{CDCl}_{3}\right) \delta$ $7.25(\mathrm{~s}, 4 \mathrm{H}), 7.20(\mathrm{~s}, 4 \mathrm{H}), 4.59(\mathrm{~s}, 4 \mathrm{H}), 4.57(\mathrm{~s}, 4 \mathrm{H}), 4.22(\mathrm{~m}, 4 \mathrm{H})$, $4.17(\mathrm{~m}, 8 \mathrm{H}), 3.97(\mathrm{~m}, 12 \mathrm{H}), 3.86(\mathrm{~m}, 8 \mathrm{H}), 3.79(\mathrm{~m}, 4 \mathrm{H}), 3.72(\mathrm{~m}$, $12 \mathrm{H}), 3.66-3.62(\mathrm{~m}, 24 \mathrm{H}), 3.54-3.51(\mathrm{~m}, 12 \mathrm{H}), 3.36(\mathrm{~s}, 6 \mathrm{H}), 3.35$ $(\mathrm{s}, 12 \mathrm{H}), 1.80(\mathrm{~m}, 8 \mathrm{H}), 1.73(\mathrm{~m}, 4 \mathrm{H}), 1.47(\mathrm{~m}, 12 \mathrm{H}), 1.28(\mathrm{~m}, 48 \mathrm{H})$, $0.89-0.86(\mathrm{~m}, 18 \mathrm{H}) .{ }^{13} \mathrm{C} \mathrm{NMR}\left(126 \mathrm{MHz}, \mathrm{CDCl}_{3}\right) \delta 165.9,165.5$, 153.2, 152.5, 143.2, 142.9, 124.3, 123.8, 109.2, 107.6, 76.1, 72.6, 72.0, $71.4,70.9,70.8,70.6$ 69.7, 69.1, 62.7, 59.1, 43.6, 40.7, 39.7, 30.6, 30.5, $29.4,29.2,23.9,23.7,23.2,23.2,14.2,14.2,11.3$. MALDI-TOF $(\mathrm{m} / z)$ : $[\mathrm{M}+\mathrm{Na}]^{+}$calcd for $\mathrm{C}_{123} \mathrm{H}_{208} \mathrm{O}_{36} \mathrm{Na}, 2316$; found, 2315 .

3,4,5-dm(rac). Purity (HPLC): $99 \%+.{ }^{1} \mathrm{H}$ NMR $\left(500 \mathrm{MHz}, \mathrm{CDCl}_{3}\right)$ $\delta 7.26(\mathrm{~s}, 4 \mathrm{H}), 7.23(\mathrm{~s}, 4 \mathrm{H}), 4.59(\mathrm{~s}, 4 \mathrm{H}), 4.58(\mathrm{~s}, 4 \mathrm{H}), 4.22(\mathrm{~m}, 4 \mathrm{H})$, $4.17(\mathrm{~m}, 8 \mathrm{H}), 4.08-3.98(\mathrm{~m}, 12 \mathrm{H}), 3.86(\mathrm{~m}, 8 \mathrm{H}), 3.79(\mathrm{~m}, 4 \mathrm{H}), 3.72$ $(\mathrm{m}, 12 \mathrm{H}), 3.66-3.62(\mathrm{~m}, 24 \mathrm{H}), 3.54-3.51(\mathrm{~m}, 12 \mathrm{H}), 3.36(\mathrm{~s}, 6 \mathrm{H})$, $3.35(\mathrm{~s}, 12 \mathrm{H}), 1.90-1.78(\mathrm{~m}, 6 \mathrm{H}), 1.70(\mathrm{~m}, 6 \mathrm{H}), 1.60-1.48$ $(\mathrm{m}, 12 \mathrm{H}), 1.37-1.23(\mathrm{~m}, 18 \mathrm{H}), 1.19-0.11(\mathrm{~m}, 18 \mathrm{H}), 0.95-0.91$ $(\mathrm{m}, 18 \mathrm{H}), 0.86(\mathrm{~m}, 36 \mathrm{H}) .{ }^{13} \mathrm{C}$ NMR $\left(126 \mathrm{MHz}, \mathrm{CDCl}_{3}\right) \delta 165.8$, 165.5, 153.0, 152.5, 143.2, 143.0, 124.3, 124.0, 109.2, 108.2, 72.6, 72.0, $71.9,70.9,70.8,70.6,69.7,69.1,67.6,62.7,62.6,59.1,43.6,39.5,39.4$, $37.6,37.6,37.5,37.5,36.5,29.9,29.8,28.1,24.8,24.8,22.8,22.7,19.6$, 19.6. MALDI-TOF $(\mathrm{m} / z)$ : $[\mathrm{M}+\mathrm{Na}]^{+}$calcd for $\mathrm{C}_{135} \mathrm{H}_{232} \mathrm{O}_{36} \mathrm{Na}$, 2484; found, 2484 .

\section{RESULTS AND DISCUSSION}

Natural Polyphenols and Phenolic Acids as Building Blocks for Programmed Synthetic Macromolecules. Natural phenols, resorcinol, pyrocatechols, pyrogallols, and phenolic acids including $\alpha$-resorcylic acid, protocatechuic acid, and gallic acid (Figure 5) are present in a variety of unrelated

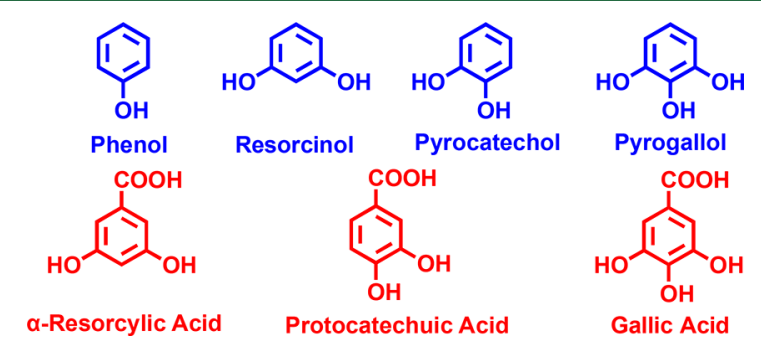

Figure 5. Natural plant phenols, polyphenols, and constitutional isomeric $\mathrm{AB}_{2}$ and $\mathrm{AB}_{3}$ phenolic acids.

types of fruits, vegetables, cereals, legumes, and their derivatives such as coffee, wine, and chocolate. ${ }^{1-7}$

Polyphenols and phenolic acids are generated as secondary metabolites during photosynthesis, and they are stored in plant and spice leaves. Their role in plant metabolism is still under investigation. It has been reported that phenolic acids act as signaling molecules, defense agents, and preservatives and are responsible for the color and taste of fruits and vegetables. They have been intensively studied for their therapeutic effect on human health. Their main natural function utilizes the antioxidant and free radical scavenger activities of these molecules (Figure 6). ${ }^{1-7}$

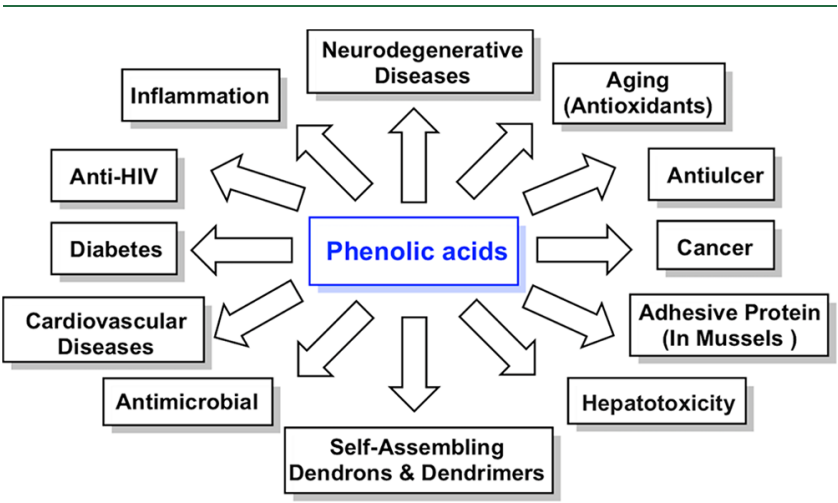

Figure 6. Functions and applications of natural, including plant, phenolic acids.

Even at low concentration, they can prevent proteins and other macromolecules from undergoing oxidative damage by direct quenching of active oxygen species, inhibition of enzymes, and chelation of metal ions. Thanks to their antioxidant activity, a diet rich in phenolic acids has beneficial effects on aging and stress-induced disorders, but they are also used as natural additives and preservatives in the food industry. They find therapeutic use for the treatment of diseases where oxidative processes play an important role, such as heart disease, arteriosclerosis, neurodegenerative diseases, and some types of cancer. Lately, they have also been employed in the treatment of diabetes mellitus and microbial infections (Figure 7). Furthermore, the

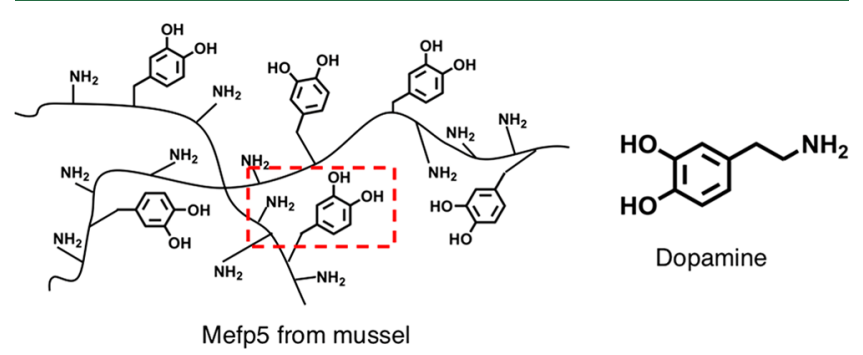

Figure 7. Schematic illustration of Mefp-5 protein from mussels and dopamine-containing amine and catechol functional groups (broken red square) used to design mussel-inspired surface coatings. ${ }^{100}$

phosphoprotein mussel foot protein Mefp-5 found in mussels exhibits very strong adhesion to surfaces due to polyphenols. Dopamine building blocks that mimic Mefp-5 protein-containing polyphenols are extensively employed to design musselinspired surface coating materials (Figure 7). ${ }^{101-104}$

Synthetic Phenolic Acids as Building Blocks for Programmed Synthetic Macromolecules. In addition to the natural phenolic acids shown in Figure 5, four additional libraries of constitutional isomeric $A_{2}$ and $A B_{3}, A_{4}$, and $A B_{5}$ phenolic acids were designed and investigated in our laboratory for the synthesis of Programmed Synthetic Macromolecules. ${ }^{25-27,29}$ Their structures are shown in Figure 8.

Synthesis of the Amphiphilic Janus Dendrimer Library. The unique properties of DSs, the facile and versatile synthesis of amphiphilic JDs, and their self-assembly have been 


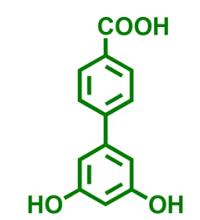<smiles>O=C(O)CCc1cc(O)cc(O)c1</smiles><smiles>O=C(O)CCc1ccc(O)c(O)c1</smiles><smiles>O=C(O)CCc1ccc(-c2cc(O)cc(O)c2)cc1</smiles><smiles>O=C(O)CCc1ccc(-c2ccc(O)c(O)c2)cc1</smiles>

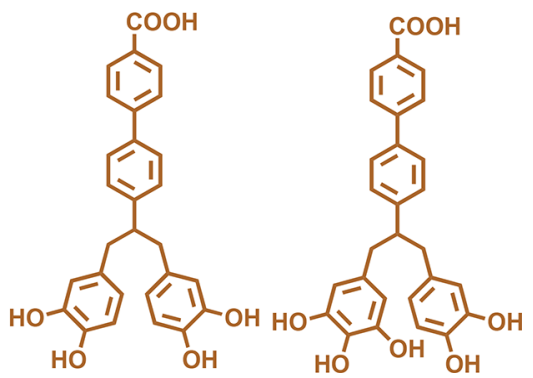

Figure 8. Synthetic constitutional isomeric $\mathrm{AB}_{2}, \mathrm{AB}_{3}, \mathrm{AB}_{4}$, and $\mathrm{AB}_{5}$ phenolic acids ${ }^{25-27,29}$ designed and employed in the Percec laboratory for the synthesis of libraries of self-assembling dendrons and dendrimers.

reported previously. ${ }^{82-86}$ The formation of biomimetic vesicles from amphiphilic building blocks follows a complex mechanism that requires additional experiments. The modular design of many different libraries of molecular building blocks will contribute to the elucidation of their mechanism of self-assembly. Size, morphology and mechanical properties of the selfassembled DSs can be predicted based on the molecular structure of JDs. ${ }^{85}$ During the formation of DSs in polar media, the substitution pattern in the first generation (G1) dendron in the hydrophobic part of JDs is determinant for the interactions between the medium and the hydrophilic part of the molecule and for the degree of interdigitation in the hydrophobic bilayer of the vesicles. For maximizing the stability and size of DSs, it has been shown that the $(3,4,5)-G 1$ substitution in the hydrophilic part containing triethylene glycol of JDs results in a better solvation of the newly formed vesicles, and the $(3,5)$-G1 pattern in the hydrophobic side allows more efficient interdigitation of the alkyl chains as well as tight packing, resulting in tougher membranes compared to those of the $(3,4)-\mathrm{G} 1$ and $(3,4,5)-\mathrm{G} 1$ patterns. A systematic synthesis of JDs with different lengths and degrees of branching of the alkyl chains in the hydrophobic dendron and screening of the effects on size and stability of DSs have not yet been performed. With this in mind, a new library of 13 amphiphilic JDs has been prepared by an accelerated iterative modular synthetic approach.

Convergent synthesis of the JDs has been performed by diversifying the structure of the alkyl chains of the (3,5)- and $(3,4,5)$-substituted hydrophobic dendrons from phenolic esters, as shown in Schemes 2 and 3. The alkylation of methyl 3,4,5trihydroxybenzoate and methyl 3,5-bihydroxybenzoate was performed with 10 different alkyl bromides. The conventional conditions for the alkylation (Scheme 2 , asterisk) are $80^{\circ} \mathrm{C}$ and $15 \mathrm{~h}$, which were adopted from the synthesis of libraries of dendrons, dendrimers, and Janus dendrimers with the phenolic acid building blocks. ${ }^{9,82}$ The alkylation reaction has been optimized according to a previous study. ${ }^{105}$ The most influential parameters affecting yield and reaction time for the alkylation of 3,4,5-trihydroxybenzoate were found to be temperature, equivalents of the base, and stirring rate. On the basis of this evaluation, an improved synthetic design has been obtained by elevating the reaction temperature from 80 to $120{ }^{\circ} \mathrm{C}$ and by increasing the concentration of reagents by $50 \%$ and the amount of base by $20 \%$. By using these optimized conditions, smaller
Scheme 2. Synthesis of 3,5-Substituted Phenolic Acids

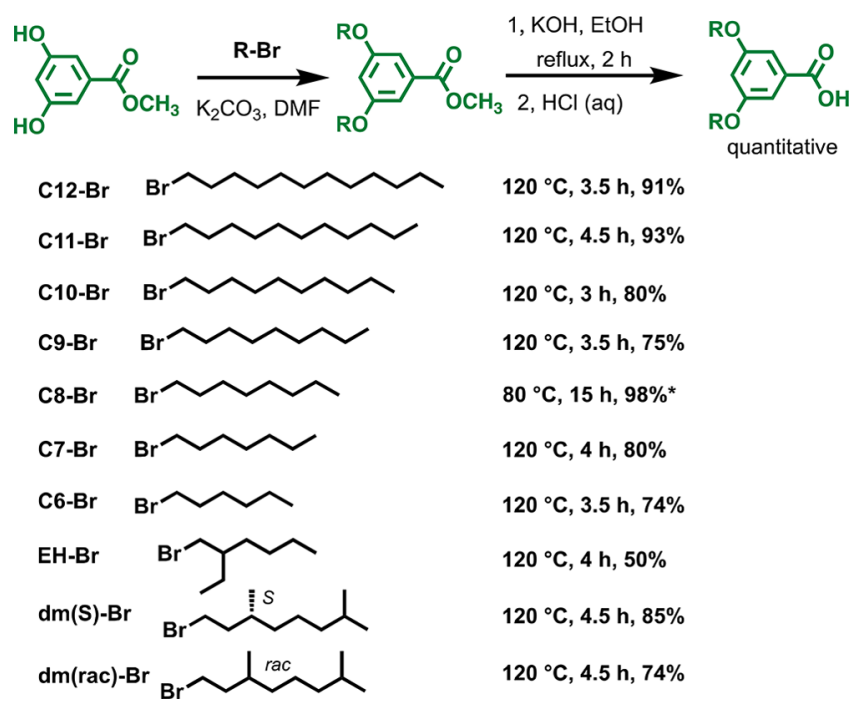

${ }^{a}$ Asterisk indicates conventional reaction temperature $\left(80{ }^{\circ} \mathrm{C}\right)$ and time $(15 \mathrm{~h})$.

Scheme 3. Synthesis of 3,4,5-Substituted Phenolic Acids

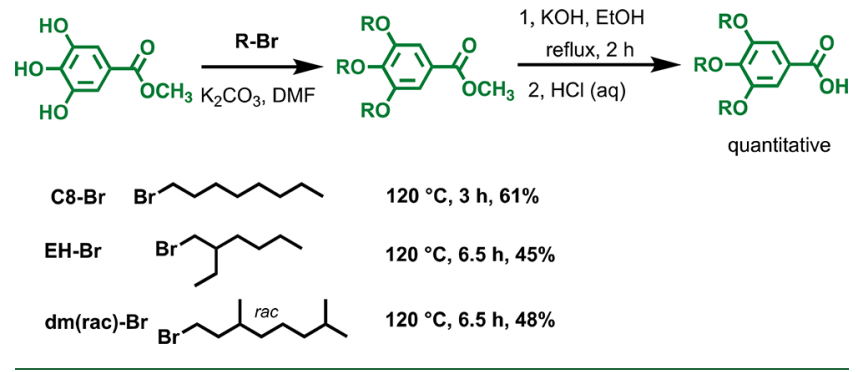

amounts of alkyl bromides could be used, but more importantly, a reduction of $\sim 70 \%$ of the reaction time was obtained. The yield of the newly optimized alkylation reactions ranged between 50 and $90 \%$. The subsequent hydrolysis of the alkyl-substituted benzoates gave the alkyl-substituted phenolic acids that were coupled to the $(3,4,5)$-substituted hydrophilic dendron (compound 6) as shown in Scheme 1.

The length of the linear alkyl chains was varied from 6 to 12 carbons. Three branched molecules were also employed: two 

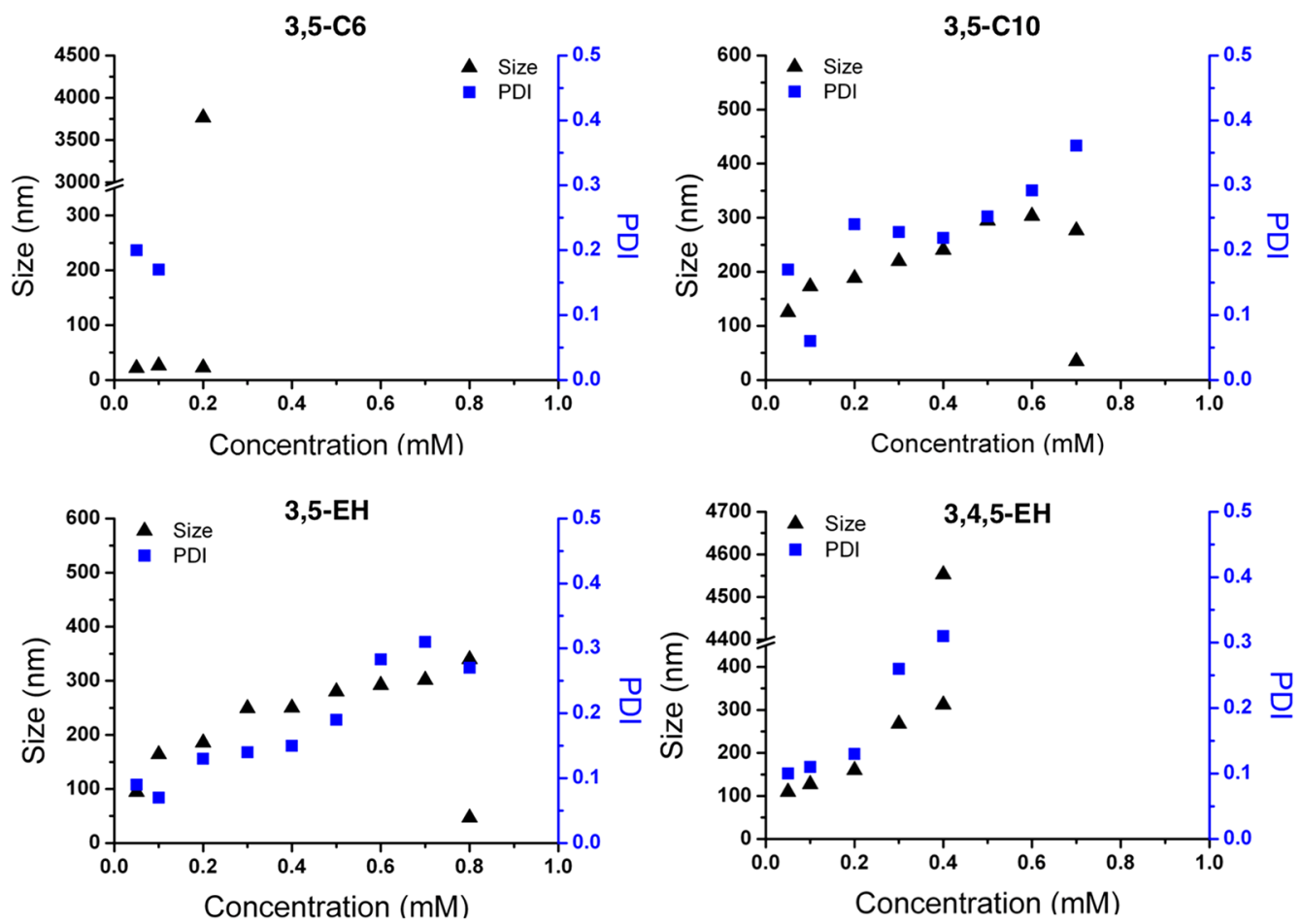

Figure 9. Representative concentration-dependent dynamic light scattering data.

being pure enantiomers and one a racemic mixture. As discussed previously, interdigitation of the alkyl chains is fundamental for the formation of robust membranes, and it is intuitive to think that a longer chain results in a more stable hydrophobic bilayer. The modulation of chain length was carried out to test the minimum number of carbons required to form stable vesicles. Furthermore, the introduction of branching points on the alkyl chains helps in understanding what happens when we introduce a degree of disruption in the packing. Biological membranes are made of phospholipids that are packed with each other by their hydrophobic chains. The presence of unsaturated bonds, stereocenters, and branching does not destabilize the membranes but are utilized in stabilization and modulation of physical properties of the membrane such as stabilization of transmembrane proteins and modulation of the crystallization of the membrane for adaptation to lower temperature environments, resulting in the diversity of structures we find in nature. ${ }^{106}$ The creation of this library of JDs is an attempt to mimic the diversity of biological membranes and to elucidate structure-property requirements needed to approach the diversity of natural membranes in JDs.

Self-Assembly of Janus Dendrimers by Injection and Screening of Dendrimersomes Size and Size Distribution vs Janus Dendrimer Concentration. The preparation of DSs by injection is by far the easiest technique to obtain stable monodisperse nanoscale vesicles. ${ }^{82,83}$ The interplay between solvation of the hydrophilic part of the JDs and the hydrophobic interactions of the alkyl chains with each other results in the formation of supramolecular assemblies whose properties depend on the molecular structure of JDs. There is an inverse proportionality between the thickness of the membrane (i.e., the interdigitation degree of the hydrophobic part of the JDs) and the size and stability of the DSs. ${ }^{85}$ The newly synthesized library of JDs was used to prepare vesicles by injection at increasing concentrations from $0.05 \mathrm{mM}$ until the stability of the DSs was compromised, resulting in bimodal size distribution.
This bimodal size distribution sets the maximum concentration that allows preparation of monodisperse DSs by injection. The formation of bimodal distributions in the DLS measurements of DSs at high concentrations is an indication of the instability of vesicles, and the result is the formation of polydisperse vesicles or large aggregates. Size and polydispersity index (PDI) of the DSs in water and buffer were monitored by DLS measurements. The effect of alkyl chain length and branching on the size and stability of DSs is shown in Figures 9 and 10 and Tables 1 and 2. The increase in length of linear alkyl chains has the effect of stabilizing the DS membrane, enabling the formation of larger vesicles at high concentrations. The self-assembly of 3,5-C6 and 3,5-C7 results in the formation of small objects that are most likely micelles rather than lamellar vesicles, suggesting that the length of the C6 and C7 chains are not enough for the stabilization of a membrane structure. There is a substantial difference in the self-assembly of 3,5-C8 and JDs having longer alkyl chains (C8-C12). The complete screening of DS size made from JDs with linear alkyl chains is reported in Table 1. The minimum chain length required to form vesicles at increasing JD concentration is C8. However, C8 generates relatively flexible and unstable vesicles. The DSs prepared from 3,5-C8, 3,5-C9, and 3,5-C10 have similar size trends and critical concentrations toward the formation of polydisperse vesicles. The introduction of one more alkyl substituent in 3,4,5-C8 does not compromise the formation of DSs even at high concentrations. However, the interdigitation of the alkyl chains becomes less efficient as evidenced by the smaller size of the vesicles compared to the DSs made from the 3,5substituted JDs. The series of DSs made from JDs with branched alkyl chains shows a different size and stability trend (Table 2). The introduction of one branching point in 3,5-EH has the effect of increasing the stabilization of the vesicles compared to those of linear 3,5-C6 and 3,5-C8 (Table 1). The substantial difference observed between 3,5-C6 and 3,5-EH may arise 


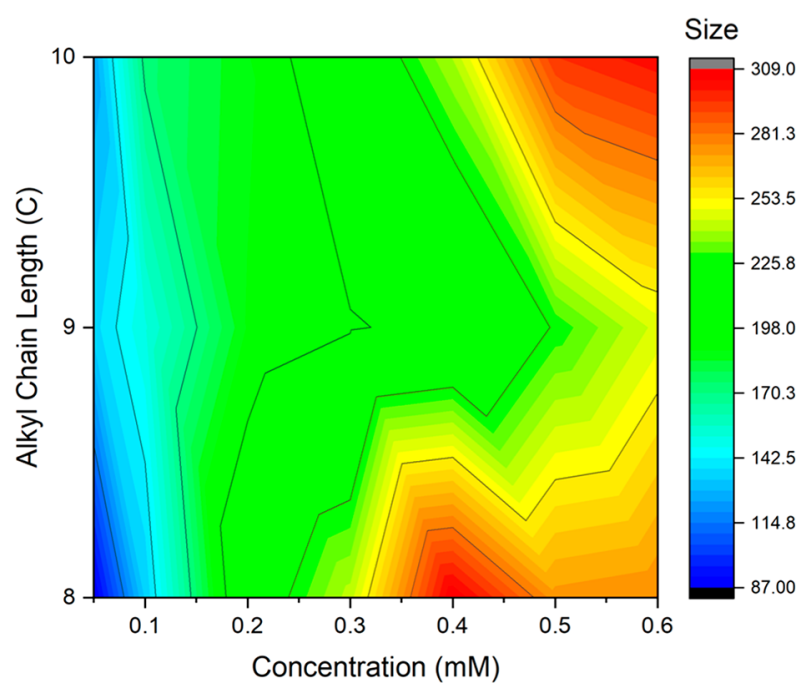

Figure 10. Contour color plots of the effect of JD concentrations and the size with the function of the chain lengths.

as a result of the increased interdigitation ability and stability of the 2-ethylhexyl compared to those of the hexyl chain in the hydrophobic bilayer of the vesicles. The size and stability increase observed in 3,5-EH is diminished when longer branched chains are used, as in 3,5-dm(S) and 3,5-dm(rac). The introduction of three branched alkyl chains in 3,4,5-EH importantly impairs the stability of vesicles at increasing JDs concentrations due to the loss in interdigitation efficiency of the alkyl chains (Figure 9 and Table 2). A possible even-odd effect of the alkyl chain length was also found in 3,5-C9 vs 3,5-C8/3,5-C10 (Figure 10).

Visualization of Dendrimersome Morphology. The visualization of DS morphology was performed by cryo-TEM on DSs prepared by injection and film hydration. Representative visualizations of monodisperse DSs prepared by injection are shown in Figure 11. Multilayer onion-like vesicles are formed from 3,4,5-EH, and mostly unilamellar vesicles are formed from 3,5-C12.

Film hydration is a slower procedure used to make giant DSs, ${ }^{82,96-99}$ which allows JDs to preassemble into two dimensionally organized bilayers on a dry Teflon surface before being hydrated with buffer solutions. It is used to prepare giant vesicles (a few micrometers); however, they are generally polydisperse, as shown in Figure 12. Most of the giant vesicles prepared and visualized by cryo-TEM show a single bilayer structure, sometimes having an elongated or irregular shape. The bilayer thicknesses were also analyzed by cryo-TEM images ${ }^{82,87}$ and summarized in Table 3. 3,5-C8 prepared by hydration resulted in vesicles that seem to have a thicker membrane $(11.5 \mathrm{~nm})$ or a nonperfect bilayer (Figure 12). This is because of their high flexibility and dynamic instability. An increasing thickness starts from the JDs with C9-C12 linear chains. For more than C8 linear chains and branched chains forming stable vesicles, the bilayer structures with clear boundaries showed thicknesses between $3.5 \mathrm{~nm}$ to maximum $9.8 \mathrm{~nm}$ (Table 3).

Giant DSs were prepared by film hydration from 3,5-C10 and $1 \%$ of rhodamine B-labeled JD 3,5-C12-RhB ${ }^{97}$ and visualized by confocal fluorescence microscopy (Figure 13). A large population of uniform giant unilamellar vesicles was observed compared to the observation on 3,5-C12 in previous publications. $^{98,99}$ This encourages us to utilize 3,5-C10 for further investigations by confocal microscopy.

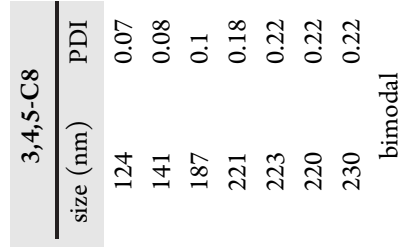

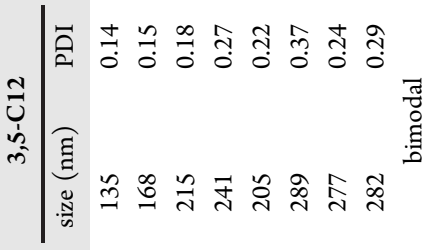
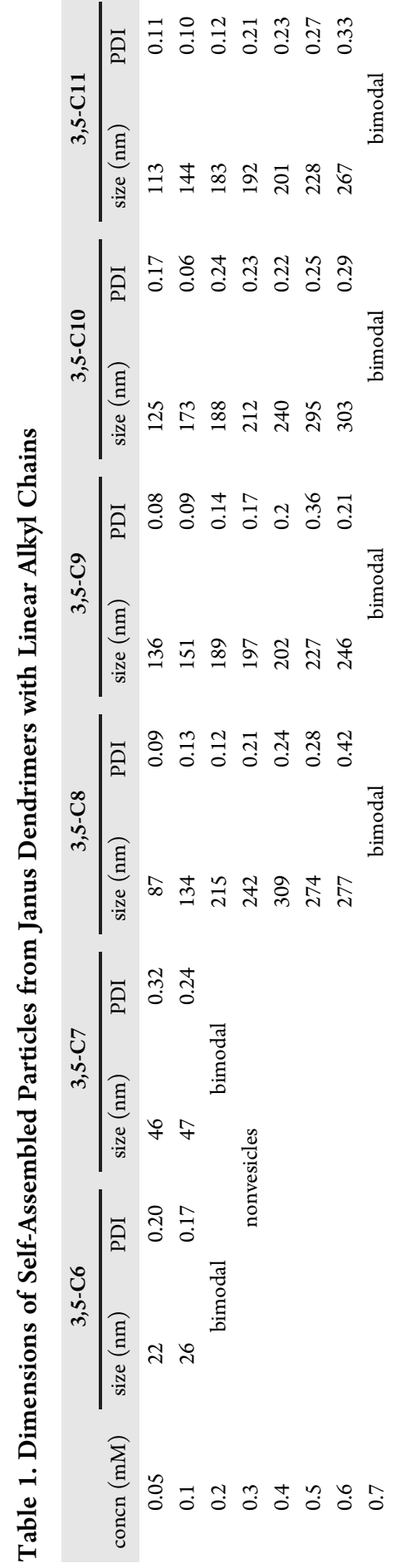
Table 2. Dimensions of Self-assembled Particles from Janus Dendrimers with Branched Alkyl Chains

\begin{tabular}{|c|c|c|c|c|c|c|c|c|c|c|}
\hline \multirow[b]{2}{*}{ concn $(\mathrm{mM})$} & \multicolumn{2}{|c|}{ 3,5-EH } & \multicolumn{2}{|c|}{$3,5-\mathrm{dm}(\mathrm{S})$} & \multicolumn{2}{|c|}{ 3,5-dm(rac) } & \multicolumn{2}{|c|}{ 3,4,5-EH } & \multicolumn{2}{|c|}{$3,4,5-\mathrm{dm}(\mathrm{rac})$} \\
\hline & size $(\mathrm{nm})$ & PDI & size $(\mathrm{nm})$ & PDI & size $(\mathrm{nm})$ & PDI & size $(\mathrm{nm})$ & PDI & size $(\mathrm{nm})$ & PDI \\
\hline 0.05 & 94 & 0.09 & 121 & 0.12 & 130 & 0.18 & 109 & 0.10 & 149 & 0.15 \\
\hline 0.1 & 164 & 0.07 & 163 & 0.14 & 160 & 0.17 & 127 & 0.11 & 175 & 0.16 \\
\hline 0.2 & 186 & 0.13 & 186 & 0.25 & 183 & 0.24 & 160 & 0.13 & 156 & 0.4 \\
\hline 0.3 & 249 & 0.14 & 208 & 0.19 & 207 & 0.22 & 268 & 0.26 & bim & \\
\hline 0.4 & 250 & 0.15 & 191 & 0.23 & 190 & 0.19 & \multicolumn{2}{|c|}{ bimodal } & & \\
\hline 0.5 & 280 & 0.19 & 236 & 0.25 & 242 & 0.29 & & & & \\
\hline 0.6 & 292 & 0.28 & \multicolumn{2}{|c|}{ bimodal } & 240 & 0.28 & & & & \\
\hline 0.7 & 302 & 0.31 & & & \multicolumn{2}{|c|}{ bimodal } & & & & \\
\hline 0.8 & \multicolumn{2}{|c|}{ bimodal } & & & & & & & & \\
\hline
\end{tabular}
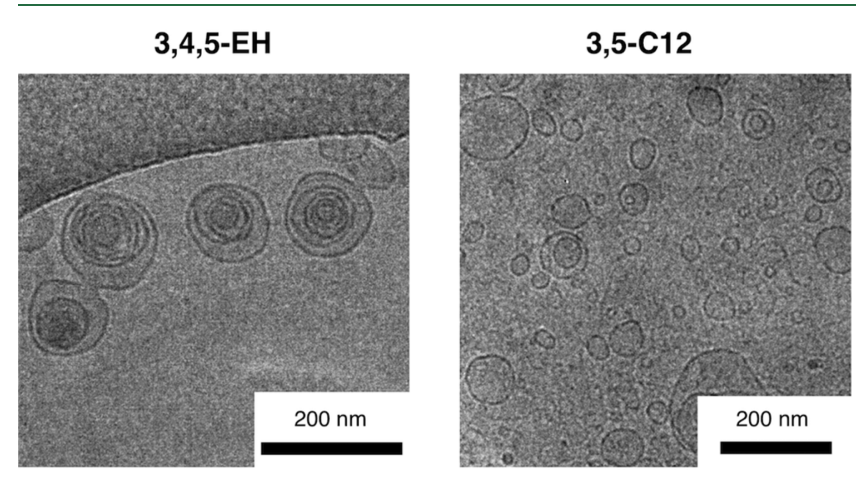

Figure 11. Representative cryo-TEM images of self-assembled structures by injection.

\section{CONCLUSIONS}

Natural and synthetic polyphenols and phenolic acids are building blocks for self-assembling dendrons and dendrimers. The discovery of self-assembling dendrons and dendrimers and their assembly into very complex structures such as FrankKasper phases evolved in additionally similar supramolecular nanostructures from block copolymers, ${ }^{107-111}$ surfactants, ${ }^{112-115}$ and other systems. ${ }^{116-119}$ Here, a library of 13 amphiphilic JDs derived from natural phenolic acids and functionalized with a variety of alkyl chains of different length and branching has been successfully synthesized by accelerated
Table 3. Average Bilayer Thickness Calculated from CryoTEM Images

\begin{tabular}{lcc} 
& av bilayer thickness $(\mathrm{nm})$ & standard deviation $(\mathrm{nm})$ \\
3,5-C6 & N/A & \\
3,5-C7 & N/A & \\
3,5-C8 & 11.5 & 2.1 \\
3,5-C9 & 3.5 & 0.4 \\
3,5-C10 & 5.6 & 0.7 \\
3,5-C12 & 5.8 & 1.0 \\
3,4,5-C8 & 5.5 & 0.2 \\
3,5-EH & 7.7 & 1.3 \\
3,5-dm(S) & 9.8 & 0.5 \\
3,5-dm(rac) & 7.7 & 0.2 \\
3,4,5-EH & 4.9 & 0.3 \\
3,4,5-dm(rac) & 6.0 & 0.5 \\
\hline
\end{tabular}

iterative methods. The alkylation of the phenolic acid derivatives methyl 3,4,5-trihydroxybenzoate and methyl 3,5-dihydroxybenzoate has been optimized resulting in a decrease of $\sim 70 \%$ of the reaction time without negatively affecting the yield. A systematic study on the size, structure, stability, and polydispersity of the self-assembled DSs was conducted at different concentrations by injection and monitored by dynamic light scattering, cryo-TEM, and confocal microscopy. The length of linear alkyl chains is fundamental for efficient formation of vesicles. For JDs substituted with linear alkyl chains in the 3,5-positions on the

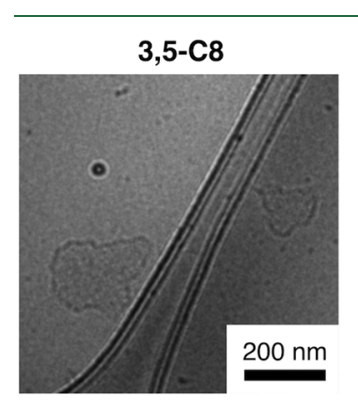

3,5-EH

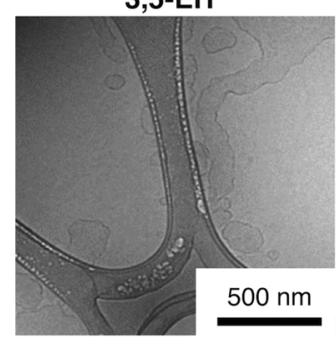

3,5-C9

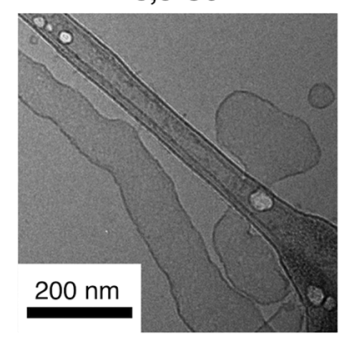

3,5-dm(S)

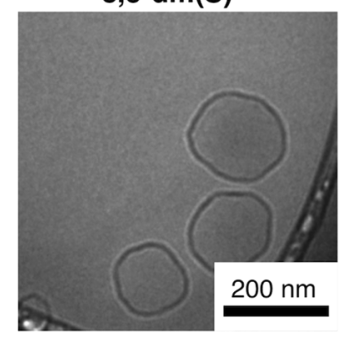

3,5-C10

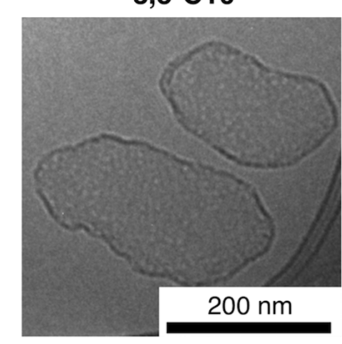

3,5-dm(rac)

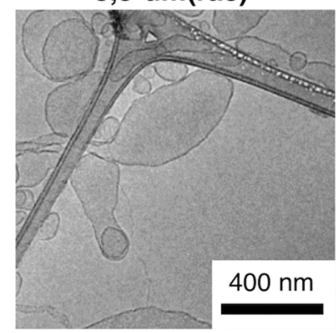

3,5-C12

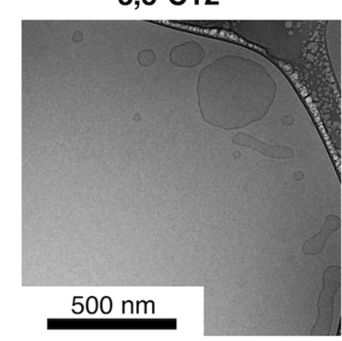

3,4,5-EH

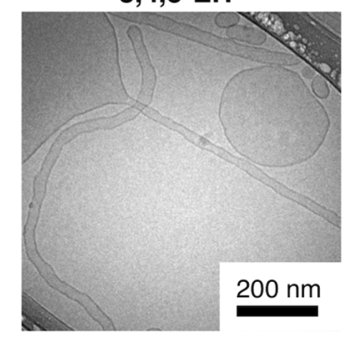

$3,4,5-\mathrm{C} 8$

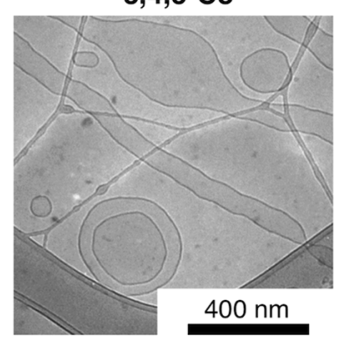

3,4,5-dm(rac)

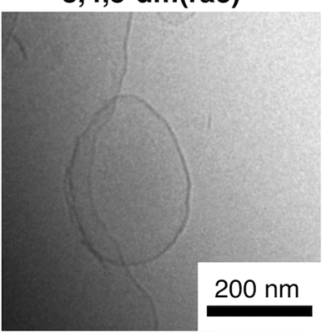

Figure 12. Representative cryo-TEM images of self-assembled structures by film hydration. 

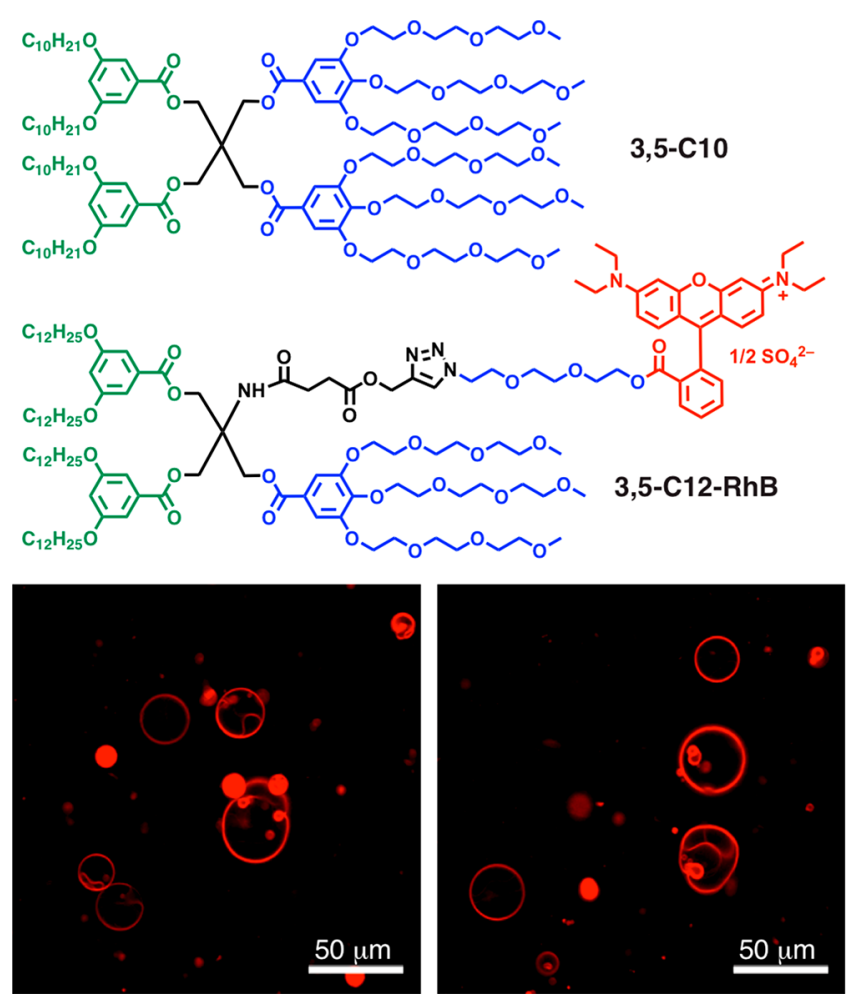

Figure 13. Representative confocal images of self-assembled structures by coassembly of 3,5-C10 with $1 \%$ of rhodamine B-labeled JD 3,5-C12RhB by film hydration in PBS.

phenolic acid part, there is a clear trend of increased size and stability of DSs above C8 with minor differences from 3,5-C9 up to 3,5-C12. C8 forms a very flexible vesicle with dynamic bilayers. Amphiphilic Janus dendrimers based on C9-C12 chains in the hydrophobic part of the JDs assemble into stable DSs in water. Their stability is predicted by the interdigitation of their alkyl chains. ${ }^{85}$ In contrast, C6 and C7 chains may not be long enough for the formation of stable vesicles based on DLS analysis and cryo-TEM measurements. These much smaller assemblies formed by 3,5-C6 and 3,5-C7 are probably micellar; $^{82}$ therefore, 3,5-C8 self-assembled into thick dynamic membrane-like structures as intermediates between micelles (C6 and C7) and vesicles (more than C8). Branched alkyl chains including 2-ethylhexyl and 3,7-dimethyloctyl chains on both 3,5- and 3,4,5-substituted phenolic acids presented stable self-assembled DSs comparable to those containing linear chains. DSs from chiral 3,5- $\mathrm{dm}(\mathrm{S})$ and racemic 3,5-dm(rac) exhibited similar size, but no onion-like assemblies were observed for 3,5-dm(S). Furthermore, the critical concentration of chiral 3,5-dm(S) is lower than that of 3,5-dm( rac) (Table 2). The self-assembled chiral/racemic DSs from 3,5-dm(S) and $3,5-\mathrm{dm}(\mathrm{rac})$ will be investigated for the role of chirality in the assembly of biological and synthetic membranes.

Among these 13 JD molecules, 3,5-C6 and 3,5-C7 form micelles, 3,5-C9, 3,5-C10, and 3,5-dm(S) form only unilamellar and stable DSs, and the other JDs show mixtures of uniand multilamellar onion-like DSs. Therefore, 3,5-C9, 3,5-C10, and $3,5-\mathbf{d m}(S)$ are recommended for experiments that require only unilamellar vesicles. With this new library of JDs and their self-assembled stable DSs, encapsulation, delivery, and change in structure during encapsulation and release are currently under investigation and will be reported in a different publication. Therefore, these JDs are expected to be utilized as important tools for nanomedicine $\mathrm{e}^{120-135}$ and synthetic cell biology.

\section{ASSOCIATED CONTENT}

\section{S Supporting Information}

The Supporting Information is available free of charge on the ACS Publications website at DOI: 10.1021/acs.biomac.8b01405.

${ }^{1} \mathrm{H}$ NMR, ${ }^{13} \mathrm{C}$ NMR, and MALDI-TOF spectra of newly synthesized Janus dendrimers and the DLS traces (PDF)

\section{AUTHOR INFORMATION}

\section{Corresponding Author}

*E-mail: percec@sas.upenn.edu.

\section{ORCID}

Qi Xiao: 0000-0002-6470-0407

Samantha E. Wilner: 0000-0002-7661-8566

Martin Möller: 0000-0002-5955-4185

Cesar Rodriguez-Emmenegger: 0000-0003-0745-0840

Tobias Baumgart: 0000-0001-7385-8460

Daniela A. Wilson: 0000-0002-8796-2274

Christopher J. Wilson: 0000-0003-0760-3421

Virgil Percec: 0000-0001-5926-0489

Notes

The authors declare no competing financial interest.

\section{ACKNOWLEDGMENTS}

This work was supported by National Science Foundation Grants DMR-1066116, DMR-1807127 (to V.P.), the P. Roy Vagelos Chair at the University of Pennsylvania (to V.P.), the Alexander von Humboldt Foundation (to N.Y.K. and V.P.), National Science Foundation Grant DMR-1120901 (to M.L.K. and V.P.), the European Union's Horizon 2020 research and innovation programme under the Marie Skłodowska-Curie Grant Agreement 642687 (to I.B.), and financial support from Nankai University (to H.H.).

\section{REFERENCES}

(1) Rice-Evans, C. A.; Miller, N. J.; Paganga, G. Structure-Antioxidant Activity Relationships of Flavonoids and Phenolic Acids. Free Radical Biol. Med. 1996, 20, 933-956.

(2) Saibabu, V.; Fatima, Z.; Khan, L. A.; Hameed, S. Therapeutic Potential of Dietary Phenolic Acids. Adv. Pharmacol. Sci. 2015, 2015, 823539.

(3) Pandey, K. B.; Rizvi, S. I. Plant Polyphenols as Dietary Antioxidants in Human Health and Disease. Oxid. Med. Cell. Longevity 2009, 2, 270-278.

(4) Natella, F.; Nardini, M.; Di Felice, M.; Scaccini, C. Benzoic and Cinnamic Acid Derivatives as Antioxidants: Structure-Activity Relation. J. Agric. Food Chem. 1999, 47, 1453-1459.

(5) Mattila, P.; Hellström, J.; Törrönen, R. Phenolic Acids in Berries, Fruits, and Beverages. J. Agric. Food Chem. 2006, 54, 7193-7199.

(6) Haminiuk, C. W. I.; Maciel, G. M.; Plata-Oviedo, M. S. V.; Peralta, R. M. Phenolic Compounds in Fruits - an Overview. Int. J. Food Sci. Technol. 2012, 47, 2023-2044.

(7) Quideau, S.; Deffieux, D.; Douat-Casassus, C.; Pouységu, L. Plant Polyphenols: Chemical Properties, Biological Activities, and Synthesis. Angew. Chem., Int. Ed. 2011, 50, 586-621.

(8) Rudick, J. G.; Percec, V. Induced Helical Backbone Conformations of Self-Organizable Dendronized Polymers. Acc. Chem. Res. 2008, 41, 1641-1652.

(9) Rosen, B. M.; Wilson, C. J.; Wilson, D. A.; Peterca, M.; Imam, M. R.; Percec, V. Dendron-Mediated Self-Assembly, Disassembly, and SelfOrganization of Complex Systems. Chem. Rev. 2009, 109, 6275-6540. 
(10) Roche, C.; Percec, V. Complex Adaptable Systems Based on SelfAssembling Dendrimers and Dendrons: Toward Dynamic Materials. Isr. J. Chem. 2013, 53, 30-44.

(11) Sun, H.-J.; Zhang, S.; Percec, V. From Structure to Function via Complex Supramolecular Dendrimer Systems. Chem. Soc. Rev. 2015, 44, 3900-3923.

(12) Percec, V. From Synthetic Macromolecules to Biological-Like Complex Systems. Adv. Polym. Sci. 2013, 261, 173-198.

(13) Cochran, W.; Crick, F. H.; Vand, V. The Structure of Synthetic Polypeptides. I. The Transform of Atoms on a Helix. Acta Crystallogr. 1952, 5, 581-586.

(14) Watson, J. D.; Crick, F. H. C. Molecular Structure of Nucleic Acids: A Structure for Deoxyribose Nucleic Acid. Nature 1953, 171, 737-738.

(15) Klug, A. From Macromolecules to Biological Assemblies (Nobel Lecture). Angew. Chem., Int. Ed. Engl. 1983, 22, 565-582.

(16) Peterca, M.; Percec, V.; Imam, M. R.; Leowanawat, P.; Morimitsu, K.; Heiney, P. A. Molecular Structure of Helical Supramolecular Dendrimers. J. Am. Chem. Soc. 2008, 130, 1484014852 .

(17) Balagurusamy, V. S. K.; Ungar, G.; Percec, V.; Johansson, G. Rational Design of the First Spherical Supramolecular Dendrimers SelfOrganized in a Novel Thermotropic Cubic Liquid-Crystalline Phase and the Determination of Their Shape by X-Ray Analysis. J. Am. Chem. Soc. 1997, 119, 1539-1555.

(18) Hudson, S. D.; Jung, H.-T.; Percec, V.; Cho, W.-D.; Johansson, G.; Ungar, G.; Balagurusamy, V. S. K. Direct Visualization of Individual Cylindrical and Spherical Supramolecular Dendrimers. Science 1997, 278, 449-452.

(19) Percec, V.; Cho, W.-D.; Mosier, P. E.; Ungar, G.; Yeardley, D. J. P. Structural Analysis of Cylindrical and Spherical Supramolecular Dendrimers Quantifies the Concept of Monodendron Shape Control by Generation Number. J. Am. Chem. Soc. 1998, 120, 11061-11070.

(20) Percec, V.; Cho, W.-D.; Möller, M.; Prokhorova, S. A.; Ungar, G.; Yeardley, D. J. P. Design and Structural Analysis of the First Spherical Monodendron Self-Organizable in a Cubic Lattice. J. Am. Chem. Soc. 2000, 122, 4249-4250.

(21) Percec, V.; Cho, W.-D.; Ungar, G. Increasing the Diameter of Cylindrical and Spherical Supramolecular Dendrimers by Decreasing the Solid Angle of Their Monodendrons via Periphery Functionalization. J. Am. Chem. Soc. 2000, 122, 10273-10281.

(22) Percec, V.; Cho, W.-D.; Ungar, G.; Yeardley, D. J. P. Synthesis and Structural Analysis of Two Constitutional Isomeric Libraries of $\mathrm{AB}_{2}$-Based Monodendrons and Supramolecular Dendrimers. J. Am. Chem. Soc. 2001, 123, 1302-1315.

(23) Percec, V.; Mitchell, C. M.; Cho, W.-D.; Uchida, S.; Glodde, M.; Ungar, G.; Zeng, X.; Liu, Y.; Balagurusamy, V. S. K.; Heiney, P. A. Designing Libraries of First Generation $\mathrm{AB}_{3}$ and $\mathrm{AB}_{2}$ Self-Assembling Dendrons via the Primary Structure Generated from Combinations of $(\mathrm{AB})_{\mathrm{Y}}-\mathrm{AB}_{3}$ and $(\mathrm{AB})_{\mathrm{Y}}-\mathrm{AB}_{2}$ Building Blocks. J. Am. Chem. Soc. 2004, 126, 6078-6094.

(24) Percec, V.; Smidrkal, J.; Peterca, M.; Mitchell, C. M.; Nummelin, S.; Dulcey, A. E.; Sienkowska, M. J.; Heiney, P. A. Self-Assembly of Hybrid Dendrons with Complex Primary Structure Into Functional Helical Pores. Chem. - Eur. J. 2007, 13, 3989-4007.

(25) Percec, V.; Holerca, M. N.; Nummelin, S.; Morrison, J. J.; Glodde, M.; Smidrkal, J.; Peterca, M.; Rosen, B. M.; Uchida, S.; Balagurusamy, V. S. K.; Sienkowska, M. J.; Heiney, P. A. Exploring and Expanding the Structural Diversity of Self-Assembling Dendrons through Combinations of $A B$, Constitutional Isomeric $\mathrm{AB}_{2}$, and $\mathrm{AB}_{3}$ Biphenyl-4-Methyl Ether Building Blocks. Chem. - Eur. J. 2006, 12, 6216-6241.

(26) Percec, V.; Peterca, M.; Sienkowska, M. J.; Ilies, M. A.; Aqad, E.; Smidrkal, J.; Heiney, P. A. Synthesis and Retrostructural Analysis of Libraries of $\mathrm{AB}_{3}$ and Constitutional Isomeric $\mathrm{AB}_{2}$ Phenylpropyl EtherBased Supramolecular Dendrimers. J. Am. Chem. Soc. 2006, 128, 33243334.

(27) Percec, V.; Won, B. C.; Peterca, M.; Heiney, P. A. Expanding the Structural Diversity of Self-Assembling Dendrons and Supramolecular
Dendrimers via Complex Building Blocks. J. Am. Chem. Soc. 2007, 129, 11265-11278.

(28) Percec, V.; Holerca, M. N.; Uchida, S.; Cho, W.-D.; Ungar, G.; Lee, Y.; Yeardley, D. J. P. Exploring and Expanding the ThreeDimensional Structural Diversity of Supramolecular Dendrimers with the Aid of Libraries of Alkali Metals of Their $\mathrm{AB}_{3}$ Minidendritic Carboxylates. Chem. - Eur. J. 2002, 8, 1106-1117.

(29) Rosen, B. M.; Wilson, D. A.; Wilson, C. J.; Peterca, M.; Won, B. C.; Huang, C.; Lipski, L. R.; Zeng, X.; Ungar, G.; Heiney, P. A.; Percec, V. Predicting the Structure of Supramolecular Dendrimers via the Analysis of Libraries of $\mathrm{AB}_{3}$ and Constitutional Isomeric $\mathrm{AB}_{2}$ Biphenylpropyl Ether Self-Assembling Dendrons. J. Am. Chem. Soc. 2009, 131, 17500-17521.

(30) Percec, V.; Ahn, C.-H.; Bera, T. K.; Ungar, G.; Yeardley, D. J. P. Coassembly of a Hexagonal Columnar Liquid Crystalline Superlattice from Polymer(s) Coated with a Three-Cylindrical Bundle Supramolecular Dendrimer. Chem. - Eur. J. 1999, 5, 1070-1083.

(31) Percec, V.; Bera, T. K.; Glodde, M.; Fu, Q.; Balagurusamy, V. S. K.; Heiney, P. A. Hierarchical Self-Assembly, Coassembly, and SelfOrganization of Novel Liquid Crystalline Lattices and Superlattices from a Twin-Tapered Dendritic Benzamide and Its Four-CylinderBundle Supramolecular Polymer. Chem. - Eur. J. 2003, 9, 921-935.

(32) Percec, V.; Ahn, C.-H.; Cho, W.-D.; Jamieson, A. M.; Kim, J.; Leman, T.; Schmidt, M.; Gerle, M.; Möller, M.; Prokhorova, S. A.; Sheiko, S. S.; Cheng, S. Z. D.; Zhang, A.; Ungar, G.; Yeardley, D. J. P. Visualizable Cylindrical Macromolecules with Controlled Stiffness from Backbones Containing Libraries of Self-Assembling Dendritic Side Groups. J. Am. Chem. Soc. 1998, 120, 8619-8631.

(33) Rosen, B. M.; Peterca, M.; Huang, C.; Zeng, X.; Ungar, G.; Percec, V. Deconstruction as a Strategy for the Design of Libraries of Self-Assembling Dendrons. Angew. Chem., Int. Ed. 2010, 49, 70027005.

(34) Dukeson, D. R.; Ungar, G.; Balagurusamy, V. S. K.; Percec, V.; Johansson, G. A.; Glodde, M. Application of Isomorphous Replacement in the Structure Determination of a Cubic Liquid Crystal Phase and Location of Counterions. J. Am. Chem. Soc. 2003, 125, 15974-15980.

(35) Ungar, G.; Liu, Y.; Zeng, X.; Percec, V.; Cho, W.-D. Giant Supramolecular Liquid Crystal Lattice. Science 2003, 299, 1208-1211.

(36) Zeng, X.; Ungar, G.; Liu, Y.; Percec, V.; Dulcey, A. E.; Hobbs, J. K. Supramolecular Dendritic Liquid Quasicrystals. Nature 2004, 428, $157-160$.

(37) Peterca, M.; Percec, V. Recasting Metal Alloy Phases with Block Copolymers. Science 2010, 330, 333-334.

(38) Ungar, G.; Percec, V.; Zeng, X.; Leowanawat, P. Liquid Quasicrystals. Isr. J. Chem. 2011, 51, 1206-1215.

(39) Percec, V.; Ahn, C.-H.; Barboiu, B. Self-Encapsulation, Acceleration and Control in the Radical Polymerization of Monodendritic Monomers via Self-Assembly. J. Am. Chem. Soc. 1997, 119, 12978-12979.

(40) Percec, V.; Ahn, C.-H.; Ungar, G.; Yeardley, D. J. P.; Möller, M.; Sheiko, S. S. Controlling Polymer Shape Through the Self-Assembly of Dendritic Side-Groups. Nature 1998, 391, 161-164.

(41) Percec, V.; Rudick, J. G.; Peterca, M.; Wagner, M.; Obata, M.; Mitchell, C. M.; Cho, W.-D.; Balagurusamy, V. S. K.; Heiney, P. A. Thermoreversible Cis-Cisoidal to Cis-Transoidal Isomerization of Helical Dendronized Polyphenylacetylenes. J. Am. Chem. Soc. 2005, 127, 15257-15264.

(42) Jishkariani, D.; MacDermaid, C. M.; Timsina, Y. N.; Grama, S.; Gillani, S. S.; Divar, M.; Yadavalli, S. S.; Moussodia, R.-O.; Leowanawat, P.; Camacho, A. M. B.; Walter, R.; Goulian, M.; Klein, M. L.; Percec, V. Self-Interrupted Synthesis of Sterically Hindered Aliphatic Polyamide Dendrimers. Proc. Natl. Acad. Sci. U. S. A. 2017, 114, E2275-E2284.

(43) Percec, V.; Glodde, M.; Bera, T. K.; Miura, Y.; Shiyanovskaya, I.; Singer, K. D.; Balagurusamy, V. S. K.; Heiney, P. A.; Schnell, I.; Rapp, A.; Spiess, H.-W.; Hudson, S. D.; Duan, H. Self-organization of Supramolecular Helical Dendrimers into Complex Electronic Materials. Nature 2002, 419, 384-387.

(44) Percec, V.; Glodde, M.; Peterca, M.; Rapp, A.; Schnell, I.; Spiess, H. W.; Bera, T. K.; Miura, Y.; Balagurusamy, V. S. K.; Aqad, E.; Heiney, 
P. A. Self-Assembly of Semifluorinated Dendrons Attached to ElectronDonor Groups Mediates Their $\pi$-Stacking via a Helical Pyramidal Column. Chem. - Eur. J. 2006, 12, 6298-6314.

(45) Percec, V.; Aqad, E.; Peterca, M.; Imam, M. R.; Glodde, M.; Bera, T. K.; Miura, Y.; Balagurusamy, V. S. K.; Ewbank, P. C.; Würthner, F.; Heiney, P. A. Self-Assembly of Semifluorinated Minidendrons Attached to Electron-Acceptor Groups into Pyramidal Columns. Chem. - Eur. J. 2007, 13, 3330-3345.

(46) Percec, V.; Dulcey, A. E.; Balagurusamy, V. S. K.; Miura, Y.; Smidrkal, J.; Peterca, M.; Nummelin, S.; Edlund, U.; Hudson, S. D.; Heiney, P. A.; Duan, H.; Magonov, S. N.; Vinogradov, S. A. Selfassembly of Amphiphilic Dendritic Dpeptides into Helical Pores. Nature 2004, 430, 764-768.

(47) Percec, V.; Dulcey, A. E.; Peterca, M.; Ilies, M.; Ladislaw, J.; Rosen, B. M.; Edlund, U.; Heiney, P. A. The Internal Structure of Helical Pores Self-Assembled from Dendritic Dipeptides Is Stereochemically Programmed and Allosterically Regulated. Angew. Chem., Int. Ed. 2005, 44, 6516-6521.

(48) Percec, V.; Dulcey, A. E.; Peterca, M.; Ilies, M.; Sienkowska, M.J.; Heiney, P. A. Programming the Internal Structure and Stability of Helical Pores Self-Assembled from Dendritic Dipeptides via the Protective Groups of the Peptide. J. Am. Chem. Soc. 2005, 127, 1790217909.

(49) Percec, V.; Dulcey, A. E.; Peterca, M.; Ilies, M.; Nummelin, S.; Sienkowska, M. J.; Heiney, P. A. Principles of Self-Assembly of Helical Pores from Dendritic Dipeptides. Proc. Natl. Acad. Sci. U. S. A. 2006, 103, 2518-2523.

(50) Peterca, M.; Percec, V.; Dulcey, A. E.; Nummelin, S.; Korey, S.; Ilies, M.; Heiney, P. A. Self-Assembly, Structural, and Retrostructural Analysis of Dendritic Dipeptide Pores Undergoing Reversible Circular to Elliptical Shape Change. J. Am. Chem. Soc. 2006, 128, 6713-6720.

(51) Percec, V.; Dulcey, A. E.; Peterca, M.; Adelman, P.; Samant, R.; Balagurusamy, V. S. K.; Heiney, P. A. Helical Pores Self-Assembled from Homochiral Dendritic Dipeptides Based on L-Tyr and Nonpolar Alpha-Amino Acids. J. Am. Chem. Soc. 2007, 129, 5992-6002.

(52) Kaucher, M. S.; Peterca, M.; Dulcey, A. E.; Kim, A. J.; Vinogradov, S. A.; Hammer, D. A.; Heiney, P. A.; Percec, V. Selective Transport of Water Mediated by Porous Dendritic Dipeptides. J. Am. Chem. Soc. 2007, 129, 11698-11699.

(53) Rosen, B. M.; Peterca, M.; Morimitsu, K.; Dulcey, A. E.; Leowanawat, P.; Resmerita, A.-M.; Imam, M. R.; Percec, V. Programming the Supramolecular Helical Polymerization of Dendritic Dipeptides via the Stereochemical Information of the Dipeptide. J. Am. Chem. Soc. 2011, 133, 5135-5151.

(54) Rosen, B. M.; Roche, C.; Percec, V. Self-Assembly of Dendritic Dipeptides as a Model of Chiral Selection in Primitive Biological Systems. Top. Curr. Chem. 2012, 333, 213-253.

(55) Rudick, J. G.; Percec, V. Helical Chirality in Dendronized Polyarylacetylenes. New J. Chem. 2007, 31, 1083-1096.

(56) Percec, V.; Rudick, J. G.; Peterca, M.; Heiney, P. A. Nanomechanical Function from Self-Organizable Dendronized Helical Polyphenylacetylenes. J. Am. Chem. Soc. 2008, 130, 7503-7508.

(57) Feringa, B. L.; Browne, W. R. Nanomechanics: Macromolecules Flex their Muscles. Nat. Nanotechnol. 2008, 3, 383.

(58) Rudick, J. G.; Percec, V. Nanomechanical Function Made Possible by Suppressing Structural Transformations of Polyarylacetylenes. Macromol. Chem. Phys. 2008, 209, 1759-1768.

(59) Andreopoulou, K. A.; Peterca, M.; Wilson, D. A.; Partridge, B. E.; Heiney, P. A.; Percec, V. Demonstrating the $8_{1}$-Helicity and Nanomechanical Function of Self-Organizable Dendronized Polymethacrylates and Polyacrylates. Macromolecules 2017, 50, 5271-5284.

(60) Percec, V.; Peterca, M.; Dulcey, A. E.; Imam, M. R.; Hudson, S. D.; Nummelin, S.; Adelman, P.; Heiney, P. A. Hollow Spherical Supramolecular Dendrimers. J. Am. Chem. Soc. 2008, 130, 1307913094.

(61) Percec, V.; Imam, M. R.; Peterca, M.; Wilson, D. A.; Graf, R.; Spiess, H. W.; Balagurusamy, V. S. K.; Heiney, P. A. Self-Assembly of Dendronized Triphenylenes into Helical Pyramidal Columns and Chiral Spheres. J. Am. Chem. Soc. 2009, 131, 7662-7677.
(62) Percec, V.; Imam, M. R.; Peterca, M.; Wilson, D. A.; Heiney, P. A. Self-Assembly of Dendritic Crowns into Chiral Supramolecular Spheres. J. Am. Chem. Soc. 2009, 131, 1294-1304.

(63) Sahoo, D.; Peterca, M.; Aqad, E.; Partridge, B. E.; Heiney, P. A.; Graf, R.; Spiess, H. W.; Zeng, X.; Percec, V. Hierarchical SelfOrganization of Perylene Bisimides into Supramolecular Spheres and Periodic Arrays Thereof. J. Am. Chem. Soc. 2016, 138, 14798-14807.

(64) Percec, V.; Peterca, M.; Tadjiev, T.; Zeng, X.; Ungar, G.; Leowanawat, P.; Aqad, E.; Imam, M. R.; Rosen, B. M.; Akbey, U.; Graf, R.; Sekharan, S.; Sebastiani, D.; Spiess, H. W.; Heiney, P. A.; Hudson, S. D. Self-Assembly of Dendronized Perylene Bisimides into Complex Helical Columns. J. Am. Chem. Soc. 2011, 133, 12197-12219.

(65) Percec, V.; Hudson, S. D.; Peterca, M.; Leowanawat, P.; Aqad, E.; Graf, R.; Spiess, H. W.; Zeng, X.; Ungar, G.; Heiney, P. A. SelfRepairing Complex Helical Columns Generated via Kinetically Controlled Self-Assembly of Dendronized Perylene Bisimides. J. Am. Chem. Soc. 2011, 133, 18479-18494.

(66) Percec, V.; Sun, H.-J.; Leowanawat, P.; Peterca, M.; Graf, R.; Spiess, H. W.; Zeng, X.; Ungar, G.; Heiney, P. A. Transformation from Kinetically into Thermodynamically Controlled Self-Organization of Complex Helical Columns with 3D Periodicity Assembled from Dendronized Perylene Bisimides. J. Am. Chem. Soc. 2013, 135, 41294148.

(67) Roche, C.; Sun, H.-J.; Prendergast, M. E.; Leowanawat, P.; Partridge, B. E.; Heiney, P. A.; Araoka, F.; Graf, R.; Spiess, H. W.; Zeng, X.; Spies, H. W.; Zeng, X.; Ungar, G.; Percec, V. Homochiral Columns Constructed by Chiral Self-Sorting During Supramolecular Helical Organization of Hat-Shaped Molecules. J. Am. Chem. Soc. 2014, 136, $7169-7185$

(68) Roche, C.; Sun, H.-J.; Leowanawat, P.; Araoka, F.; Partridge, B. E.; Peterca, M.; Wilson, D. A.; Prendergast, M. E.; Heiney, P. A.; Graf, R.; Spiess, H. W.; Zeng, X.; Ungar, G.; Percec, V. A Supramolecular Helix That Disregards Chirality. Nat. Chem. 2016, 8, 80-89.

(69) Peterca, M.; Imam, M. R.; Hudson, S. D.; Partridge, B. E.; Sahoo, D.; Heiney, P. A.; Klein, M. L.; Percec, V. Complex Arrangement of Orthogonal Nanoscale Columns via a Supramolecular Orientational Memory Effect. ACS Nano 2016, 10, 10480-10488.

(70) Sahoo, D.; Peterca, M.; Aqad, E.; Partridge, B. E.; Heiney, P. A.; Graf, R.; Spiess, H. W.; Zeng, X.; Percec, V. Tetrahedral Arrangements of Perylene Bisimide Columns via Supramolecular Orientational Memory. ACS Nano 2017, 11, 983-991.

(71) Sahoo, D.; Peterca, M.; Aqad, E.; Partridge, B. E.; Klein, M. L.; Percec, V. Losing Supramolecular Orientational Memory via SelfOrganization of a Misfolded Secondary Structure. Polym. Chem. 2018, 9, 2370-2381.

(72) Singer, S. J.; Nicolson, G. L. The Fluid Mosaic Model of the Structure of Cell Membranes. Science 1972, 175, 720-731.

(73) Simons, K.; Ikonen, E. Functional Rafts in Cell Membraneds. Nature 1997, 387, 569-572.

(74) Lingwood, D.; Simons, K. Lipid Rafts as a Membrane-Organizing Principle. Science 2010, 327, 46-50.

(75) Eggeling, C.; Ringemann, C.; Medda, R.; Schwarzmann, G.; Sandhoff, K.; Polyakova, S.; Belov, V. N.; Hein, B.; von Middendorff, C.; Schönle, A.; Hell, S. W. Direct Observation of the Nanoscale Dynamics of Membrane Lipids in a Living Cell. Nature 2009, 457, $1159-1162$.

(76) Sezgin, E.; Levental, I.; Mayor, S.; Eggeling, C. The Mystery of Membrane Organization: Composition, Regulation and Roles of Lipid Rafts. Nat. Rev. Mol. Cell Biol. 2017, 18, 361-374.

(77) Bangham, A. D.; Standish, M. M.; Watkins, J. C. Diffusion of Univalent Ions Across the Lamellae of Swollen Phospholipids. J. Mol. Biol. 1965, 13, 238-252.

(78) Allen, T. M.; Chonn, A. Large Unilamellar Liposomes with Low Uptake into the Reticuloendothelial System. FEBS Lett. 1987, 223, 4246.

(79) Allen, T. M.; Cullis, P. R. Drug Delivery Systems: Entering the Mainstream. Science 2004, 303, 1818-1822.

(80) Torchilin, V. P. Recent Advances with Liposomes as Pharmaceutical Carriers. Nat. Rev. Drug Discovery 2005, 4, 145-160. 
(81) Discher, B. M.; Won, Y.-Y.; Ege, D. S.; Lee, J. C.-M.; Bates, F. S.; Discher, D. E.; Hammer, D. A. Polymersomes: Tough Vesicles Made from Diblock Copolymers. Science 1999, 284, 1143-1146.

(82) Percec, V.; Wilson, D. A.; Leowanawat, P.; Wilson, C. J.; Hughes, A. D.; Kaucher, M. S.; Hammer, D. A.; Levine, D. H.; Kim, A. J.; Bates, F. S.; Davis, K. P.; Lodge, T. P.; Klein, M. L.; DeVane, R. H.; Aqad, E.; Rosen, B. M.; Argintaru, A. O.; Sienkowska, M. J.; Rissanen, K.; Nummelin, S.; Ropponen, J. Self-assembly of Janus Dendrimers into Uniform Dendrimersomes and other Complex Architectures. Science 2010, 328, 1009-1014.

(83) Sherman, S. E.; Xiao, Q.; Percec, V. Mimicking Complex Biological Membranes and Their Programmable Glycan Ligands with Dendrimersomes and Glycodendrimersomes. Chem. Rev. 2017, 117, 6538-6631.

(84) Zhang, S.; Sun, H.-J.; Hughes, A. D.; Moussodia, R.-O.; Bertin, A.; Chen, Y.; Pochan, D. J.; Heiney, P. A.; Klein, M. L.; Percec, V. SelfAssembly of Amphiphilic Janus Dendrimers into Uniform Onion-like Dendrimersomes with Predictable Size and Number of Bilayers. Proc. Natl. Acad. Sci. U. S. A. 2014, 111, 9058-9063.

(85) Peterca, M.; Percec, V.; Leowanawat, P.; Bertin, A. Predicting the Size and Properties of Dendrimersomes from the Lamellar Structure of Their Amphiphilic Janus Dendrimers. J. Am. Chem. Soc. 2011, 133, 20507-20520.

(86) Zhang, S.; Sun, H.-J.; Hughes, A. D.; Draghici, B.; Lejnieks, J.; Leowanawat, P.; Bertin, A.; Otero De Leon, L.; Kulikov, O. V.; Chen, Y.; Pochan, D. J.; Heiney, P. A.; Percec, V. Single-Single" Amphiphilic Janus Dendrimers Self-Assemble into Uniform Dendrimersomes with Predictable Size. ACS Nano 2014, 8, 1554-1565.

(87) Percec, V.; Leowanawat, P.; Sun, H.-J.; Kulikov, O.; Nusbaum, C. D.; Tran, T. M.; Bertin, A.; Wilson, D. A.; Peterca, M.; Zhang, S.; Kamat, N. P.; Vargo, K.; Moock, D.; Johnston, E. D.; Hammer, D. A.; Pochan, D. J.; Chen, Y.; Chabre, Y. M.; Shiao, T. C.; Bergeron-Brlek, M.; André, S.; Roy, R.; Gabius, H.-J.; Heiney, P. A. Modular Synthesis of Amphiphilic Janus Glycodendrimers and Their Self-Assembly into Glycodendrimersomes and Other Complex Architectures with Bioactivity to Biomedically Relevant Lectins. J. Am. Chem. Soc. 2013, 135, 9055-9077.

(88) Zhang, S.; Moussodia, R.-O.; Sun, H.-J.; Leowanawat, P.; Muncan, A.; Nusbaum, C. D.; Chelling, K. M.; Heiney, P. A.; Klein, M. L.; André, S.; Roy, R.; Gabius, H.-J.; Percec, V. Mimicking Biological Membranes with Programmable Glycan Ligands Self-Assembled from Amphiphilic Janus Glycodendrimers. Angew. Chem., Int. Ed. 2014, 53, 10899-10903.

(89) Zhang, S.; Xiao, Q.; Sherman, S. E.; Muncan, A.; Ramos Vicente, A. D. M.; Wang, Z.; Hammer, D. A.; Williams, D.; Chen, Y.; Pochan, D. J.; Vértesy, S.; André, S.; Klein, M. L.; Gabius, H.-J.; Percec, V. Glycodendrimersomes from Sequence-Defined Janus Glycodendrimers Reveal High Activity and Sensor Capacity for the Agglutination by Natural Variants of Human Lectins. J. Am. Chem. Soc. 2015, 137, 13334-13344.

(90) Xiao, Q.; Zhang, S.; Wang, Z.; Sherman, S. E.; Moussodia, R.-O.; Peterca, M.; Muncan, A.; Williams, D. R.; Hammer, D. A.; Vértesy, S.; André, S.; Gabius, H.-J.; Klein, M. L.; Percec, V. Onion-Like Glycodendrimersomes from Sequence-Defined Janus Glycodendrimers and Influence of Architecture on Reactivity to a Lectin. Proc. Natl. Acad. Sci. U. S. A. 2016, 113, 1162-1167.

(91) Zhang, S.; Moussodia, R.-O.; Murzeau, C.; Sun, H.-J.; Klein, M. L.; Vértesy, S.; André, S.; Roy, R.; Gabius, H.-J.; Percec, V. Dissecting Molecular Aspects of Cell Interactions Using Glycodendrimersomes with Programmable Glycan Presentation and Engineered Human Lectins. Angew. Chem., Int. Ed. 2015, 54, 4036-4040.

(92) Zhang, S.; Moussodia, R.-O.; Vértesy, S.; André, S.; Klein, M. L.; Gabius, H.-J.; Percec, V. Unraveling Functional Significance of Natural Variations of a Human Galectin by Glycodendrimersomes with Programmable Glycan Surface. Proc. Natl. Acad. Sci. U. S. A. 2015, $112,5585-5590$.

(93) Xiao, Q.; Wang, Z.; Williams, D.; Leowanawat, P.; Peterca, M.; Sherman, S. E.; Zhang, S.; Hammer, D. A.; Heiney, P. A.; King, S. R.; Markovitz, D. M.; André, S.; Gabius, H.-J.; Klein, M. L.; Percec, V. Why
Do Membranes of Some Unhealthy Cells Adopt a Cubic Architecture? ACS Cent. Sci. 2016, 2, 943-953.

(94) Kopitz, J.; Xiao, Q.; Ludwig, A.-K.; Romero, A.; Michalak, M.; Sherman, S. E.; Zhou, X.; Dazen, C.; Vértesy, S.; Kaltner, H.; Klein, M. L.; Gabius, H.-J.; Percec, V. Reaction of a Programmable Glycan Presentation of Glycodendrimersomes and Cells with Engineered Human Lectins To Show the Sugar Functionality of the Cell Surface. Angew. Chem., Int. Ed. 2017, 56, 14677-14681.

(95) Xiao, Q.; Ludwig, A.-K.; Romanò, C.; Buzzacchera, I.; Sherman, S. E.; Vetro, M.; Kaltner, K.; Reed, E. H.; Möller, M.; Wilson, C. J.; Hammer, D. A.; Oscarson, S.; Klein, M. L.; Gabius, H.-J.; Percec, V. Exploring Functional Pairing Between Surface Glycoconjugates and Human Galectins using Programmable Glycodendrimersomes. Proc. Natl. Acad. Sci. U. S. A. 2018, 115, E2509-E2518.

(96) Xiao, Q.; Yadavalli, S. S.; Zhang, S. D.; Sherman, S. E.; Fiorin, E.; da Silva, L.; Wilson, D. A.; Hammer, D. A.; Andre, S.; Gabius, H. J.; Klein, M. L.; Goulian, M.; Percec, V. Bioactive Cell-Like Hybrids Coassembled from (Glyco)Dendrimersomes with Bacterial Membranes. Proc. Natl. Acad. Sci. U. S. A. 2016, 113, E1134-E1141.

(97) Xiao, Q.; Rubien, J. D.; Wang, Z.; Reed, E. H.; Hammer, D. A.; Sahoo, D.; Heiney, P. A.; Yadavalli, S. S.; Goulian, M.; Wilner, S. E.; Baumgart, T.; Vinogradov, S. A.; Klein, M. L.; Percec, V. Self-Sorting and Coassembly of Fluorinated, Hydrogenated, and Hybrid Janus Dendrimers into Dendrimersomes. J. Am. Chem. Soc. 2016, 138, 12655-12663.

(98) Xiao, Q.; Sherman, S. E.; Wilner, S. E.; Zhou, X.; Dazen, C.; Baumgart, T.; Reed, E. H.; Hammer, D. A.; Shinoda, W.; Klein, M. L.; Percec, V. Janus Dendrimersomes Coassembled from Fluorinated, Hydrogenated, and Hybrid Janus Dendrimers as Models for Cell Fusion and Fission. Proc. Natl. Acad. Sci. U. S. A. 2017, 114, E7045E7053.

(99) Wilner, S. E.; Xiao, Q.; Graber, Z. T.; Sherman, S. E.; Percec, V.; Baumgart, T. Dendrimersomes Exhibit Lamellar-to-Sponge Phase Transitions. Langmuir 2018, 34, 5527-5534.

(100) Moore, J. S.; Stupp, S. I. Room Temperature Polyesterification. Macromolecules 1990, 23, 65-70.

(101) Lee, H.; Dellatore, S. M.; Miller, W. M.; Messersmith, P. B. Mussel-Inspired Surface Chemistry for Multifunctional Coatings. Science 2007, 318, 426-430.

(102) Lee, H.; Lee, B. P.; Messersmith, P. B. A Reversible Wet/Dry Adhesive Inspired by Mussels and Geckos. Nature 2007, 448, 338-341.

(103) Lee, H.; Rho, J.; Messersmith, P. B. Facile Conjugation of Biomolecules onto Surfaces via Mussel Adhesive Protein Inspired Coatings. Adv. Mater. 2009, 21, 431-434.

(104) Lee, B. P.; Messersmith, P. B.; Israelachvili, J. N.; Waite, J. H. Mussel-Inspired Adhesives and Coatings. Annu. Rev. Mater. Res. 2011, $41,99-132$.

(105) Hersmis, M. C.; Spiering, A. J. H.; Waterval, R. J. M.; Meuldijk, J.; Vekemans, J. A. J. M.; Hulshof, L. A. 3,4,5-Tri-dodecyloxybenzoic Acid: Optimisation and Scale-Up of the Synthesis. Org. Process Res. Dev. 2001, 5, 54-60.

(106) Tu, Y.; Peng, F.; Adawy, A.; Men, Y.; Abdelmohsen, L. K. E. A.; Wilson, D. A. Mimicking the Cell: Bio-Inspired Functions of Supramolecular Assemblies. Chem. Rev. 2016, 116, 2023-2078.

(107) Lee, S.; Bluemle, M. J.; Bates, F. S. Discovery of a Frank-Kasper $\sigma$-Phase in Sphere-Forming Block Copolymer Melts. Science 2010, 330, 349-353.

(108) Bates, F. S.; Hillmyer, M. A.; Lodge, T. P.; Bates, C. M.; Delaney, K. T.; Fredrickson, G. H. Multiblock Polymers: Panacea or Pandora's Box? Science 2012, 336, 434-440.

(109) Gillard, T. M.; Lee, S.; Bates, F. S. Dodecagonal Quasicrystalline order in a Diblock Copolymer melt. Proc. Natl. Acad. Sci. U. S. A. 2016, $113,5167-5172$.

(110) Chanpuriya, S.; Kim, K.; Zhang, J.; Lee, S.; Arora, A.; Dorfman, K. D.; Delaney, K. T.; Fredrickson, G. H.; Bates, F. S. Cornucopia of Nanoscale Ordered Phases in Sphere-Forming Tetrablock Terpolymers. ACS Nano 2016, 10, 4961-4972. 
(111) Kim, K.; Schulze, M. W.; Arora, A.; Lewis, R. M., III; Hillmyer, M. A.; Dorfman, K. D.; Bates, F. S. Thermal Processing of Diblock Copolymer Melts Mimics Metallurgy. Science 2017, 356, 520-523.

(112) Perroni, D. V.; Mahanthappa, M. K. Inverse Pm3n Cubic Micellar Lyotropic Phases from Zwitterionic Triazolium Gemini Surfactants. Soft Matter 2013, 9, 7919-7922.

(113) Yue, K.; Huang, M.; Marson, R. L.; He, J.; Huang, J.; Zhou, Z.; Wang, J.; Liu, C.; Yan, X.; Wu, K.; Guo, Z.; Liu, H.; Zhang, W.; Ni, P.; Wesdemiotis, C.; Zhang, W.-B.; Glotzer, S. C.; Cheng, S. Z. D. Geometry Induced Sequence of Nanoscale Frank-Kasper and Quasicrystal Mesophases in Giant Surfactants. Proc. Natl. Acad. Sci. U. S. A. 2016, 113, 14195-14200.

(114) Kim, S. A.; Jeong, K.-J.; Yethiraj, A.; Mahanthappa, M. K. LowSymmetry Sphere Packings of Simple Surfactant Micelles Induced by Ionic Sphericity. Proc. Natl. Acad. Sci. U. S. A. 2017, 114, 4072-4077.

(115) Baez-Cotto, C. M.; Mahanthappa, M. K. Micellar Mimicry of Intermetallic C14 and C15 Laves Phases by Aqueous Lyotropic SelfAssembly. ACS Nano 2018, 12, 3226-3234.

(116) Huang, M.; Hsu, C.-H.; Wang, J.; Mei, S.; Dong, X.; Li, Y.; Li, M.; Liu, H.; Zhang, W.; Aida, T.; Zhang, W.-B.; Yue, K.; Cheng, S. Z. D. Selective Assemblies of Giant Tetrahedra via Precisely Controlled Positional Interactions. Science 2015, 348, 424-428.

(117) Zhang, W.; Huang, M.; Su, H.; Zhang, S.; Yue, K.; Dong, X.-H.; Li, X.; Liu, H.; Zhang, S.; Wesdemiotis, C.; Lotz, B.; Zhang, W.-B.; Li, Y.; Cheng, S. Z. D. Toward Controlled Hierarchical Heterogeneities in Giant Molecules with Precisely Arranged Nano Building Blocks. ACS Cent. Sci. 2016, 2, 48-54.

(118) Zhang, W.; Lu, X.; Mao, J.; Hsu, C.-H.; Mu, G.; Huang, M.; Guo, Q.; Liu, H.; Wesdemiotis, C.; Li, T.; Zhang, W.-B.; Li, Y.; Cheng, S. Z. D. Sequence-Mandated, Distinct Assembly of Giant Molecules. Angew. Chem., Int. Ed. 2017, 56, 15014-15019.

(119) Ye, X.; Chen, J.; Irrgang, M. E.; Engel, M.; Dong, A.; Glotzer, S. C.; Murray, C. B. Quasicrystalline Nanocrystal Superlattice with Partial Matching Rules. Nat. Mater. 2017, 16, 214-219.

(120) Guo, X.; Szoka, F. C. Chemical Approaches to Triggerable Lipid Vesicles for Drug and Gene Delivery. Acc. Chem. Res. 2003, 36, 335341.

(121) Lee, C. C.; MacKay, J. A.; Fréchet, J. M. J.; Szoka, F. C. Designing Dendrimers for Biological Applications. Nat. Biotechnol. 2005, 23, 1517-1526.

(122) Farokhzad, O. C.; Langer, R. Impact of Nanotechnology on Drug Delivery. ACS Nano 2009, 3, 16-20.

(123) Menjoge, A. R.; Kannan, R. M.; Tomalia, D. A. Drug Discovery Today 2010, 15, 171-185.

(124) Deming, T. J. Preparation and Development of Block Copolypeptide Vesicles and Hydrogels for Biological and Medical Applications. WIREs Nanomed. Nanobiotechnol. 2014, 6, 283-297.

(125) Yu, T.; Liu, X.; Bolcato-Bellemin, A.-L.; Wang, Y.; Liu, C.; Erbacher, P.; Qu, F.; Rocchi, P.; Behr, J.-P.; Peng, L. Anamphiphilic dendrimer for effective delivery of small interfering RNA and gene silencing in vitro and in vivo. Angew. Chem., Int. Ed. 2012, 51, 84788484.

(126) Liu, X.; Zhou, J.; Yu, T.; Chen, C.; Cheng, Q.; Sengupta, K.; Huang, Y.; Li, H.; Liu, C.; Wang, Y.; Posocco, P.; Wang, M.; Cui, Q.; Giorgio, S.; Fermeglia, M.; Qu, F.; Pricl, S.; Shi, Y.; Liang, Z.; Rocchi, P.; Rossi, J.; Peng, L. Adaptive amphiphilic dendrimer-based nanoassemblies as robust and versatile siRNA delivery systems. Angew. Chem., Int. Ed. 2014, 53, 11822-11827.

(127) Wei, T.; Chen, C.; Liu, J.; Liu, C.; Posocco, P.; Liu, X.; Cheng, Q.; Huo, S.; Liang, Z.; Fermeglia, M.; Pricl, S.; Liang, X.-J.; Rocchi, P.; Peng, L. Anticancer Drug Nanomicelles Formed by Self-Assembling Amphiphilic Dendrimer to Combat Cancer Drug Resistance. Proc. Natl. Acad. Sci. U. S. A. 2015, 112, 2978-2983.

(128) Thota, B. N.S.; Urner, L. H.; Haag, R. Supramolecular Architectures of Dendritic Amphiphiles in Water. Chem. Rev. 2016, 116, 2079-2102.

(129) Filippi, M.; Catanzaro, V.; Patrucco, D.; Botta, M.; Tei, L.; Terreno, E. First in Vivo MRI Study on Theranostic Dendrimersomes. J. Controlled Release 2017, 248, 45-52.
(130) Nummelin, S.; Selin, M.; Legrand, S.; Ropponen, J.; Seitsonen, J.; Nykanen, A.; Koivisto, J.; Hirvonen, J.; Kostiainen, M. A.; Bimbo, L. M. Modular Synthesis of Self-Assembling Janus-Dendrimers and Facile Preparation of Drug-Loaded Dendrimersomes. Nanoscale 2017, 9, 7189-7198.

(131) Selin, M.; Nummelin, S.; Deleu, J.; Ropponen, J.; Viitala, T.; Lahtinen, M.; Koivisto, J.; Hirvonen, J.; Peltonen, L.; Kostiainen, M. A.; Bimbo, L. M. High-Generation Amphiphilic Janus-Dendrimers as Stabilizing Agents for Drug Suspensions. Biomacromolecules 2018, 19, 3983-3993.

(132) Yang, Y.-L.; Sheng, Y.-J.; Tsao, H.-K. Branching Pattern Effect and Co-Assembly with Lipids of Amphiphilic Janus Dendrimersomes. Phys. Chem. Chem. Phys. 2018, DOI: 10.1039/C8CP05268A.

(133) Elizondo-García, M.; Márquez-Miranda, V.; Araya-Durán, I.; Valencia-Gallegos, J.; González-Nilo, F.; Elizondo-García, M. E.; Márquez-Miranda, V.; Araya-Durán, I.; Valencia-Gallegos, J. A.; González-Nilo, F. D. Self-Assembly Behavior of Amphiphilic Janus Dendrimers in Water: A Combined Experimental and Coarse-Grained Molecular Dynamics Simulation Approach. Molecules 2018, 23, 969.

(134) Liu, X.; Gitsov, I. Thermosensitive Amphiphilic Janus Dendrimers with Embedded Metal Binding Sites. Synthesis and SelfAssembly. Macromolecules 2018, 51, 5085-5100.

(135) Taabache, S.; Bertin, A.; Taabache, S.; Bertin, A. Vesicles from Amphiphilic Dumbbells and Janus Dendrimers: Bioinspired SelfAssembled Structures for Biomedical Applications. Polymers 2017, 9, 280. 